Anna Geis/Harald Müller/Niklas Schörnig

\title{
Liberale Demokratien und Krieg
}

Warum manche kämpfen und andere nicht. Ergebnisse einer vergleichenden Inhaltsanalyse von Parlamentsdebatten

Dieser Beitrag beleuchtet zwei vernachlässigte Felder der Forschung zum Demokratischen Frieden: Mit welchen Rechtfertigungen ziehen liberale Demokratien, die untereinander so friedlich sind, gegen Nichtdemokratien in den Krieg? Wie lässt sich die variierende (Nicht-)Teilnahme von Demokratien an Kriegen erklären? Auf Basis einer sozial-konstruktivistischen Analyseperspektive präsentiert der Beitrag ausgewählte Ergebnisse einer vergleichenden inhaltsanalytischen Auswertung von Parlamentsdebatten in Frankreich, Großbritannien, Kanada, Schweden, Australien, Deutschland und den USA über jeweils drei Kriegsentscheidungen (Golfkrieg 1991, Kosovokrieg 1999, Irakkrieg 2003). Es werden Makroresultate für alle sieben Fälle vorgestellt und in der Detailanalyse Resultate für vier Länder erläutert. In der Erfassung der jeweiligen Argumentationsstrukturen sind dabei nicht nur Unterschiede zwischen den Ländern, sondern auch demokratiespezifische bzw. dem Liberalismus inhärente Gründe einer Kriegsbeteiligung von besonderem Interesse. Es wird diskutiert, inwieweit es sich bei diesen Kriegen um "demokratische Kriege" handelt und welche Konsequenzen sich hieraus für die Theorie des Demokratischen Friedens ergeben.

1. Einleitung: Der liberale Interventionismus als die Kehrseite des demokratischen Friedens

Konsolidierte Demokratien führen keine Kriege gegeneinander, sind aber bereit, gegenüber Nichtdemokratien zu den Waffen zu greifen - das ist der »Doppelbefund « der Theorien vom Demokratischen Frieden (DF). Während die Literatur über den Frieden zwischen den Demokratien kaum noch überschaubar ist, wird die Kehrseite des demokratischen Friedens, die Militanz gegenüber Nichtdemokratien, vergleichsweise selten beleuchtet. Dies ist umso bedenklicher, als die Selbstaufklärung demokratischer Gesellschaften über die eigene Kriegsneigung praxeologisch noch wichtiger ist als das Selbstlob über die eigene Friedlichkeit, das politisch bereits in fataler Weise instrumentalisiert wurde (Ish-Shalom 2006; Smith 2007: Kap. 4). Zudem vernachlässigte die Forschung zum Demokratischen Frieden die empirisch sehr unterschiedliche Kriegsneigung von Demokratien: Manche beteiligen sich sehr häufig an militärischen Kampfeinsätzen, andere seltener und wiederum andere nie (Müller 2004: 494-497; Chojnacki 2006: 21). 
Dieser Artikel ${ }^{1}$ soll einen Teil zur Beantwortung dieser miteinander verbundenen Fragen beitragen: Warum setzt sich die Friedlichkeit der Demokratien gegenüber Nichtdemokratien nicht durch? Und wie lässt sich das unterschiedliche Verhalten von Demokratien erklären? Es geht dabei nicht um die Frage, warum Demokratien in manchen Konflikten intervenieren, in anderen gar nicht (z.B. Ruanda), sondern auf welcher Begründungsbasis - wenn denn Demokratien Krieg führen - manche sich beteiligen und andere nicht. Die beiden Leitfragen können hier selbstverständlich nicht umfassend abgehandelt werden, jedoch soll eine vergleichende inhaltsanalytische Auswertung von parlamentarischen Vorkriegsdiskursen in sieben westlichen Demokratien (Australien, Deutschland, Frankreich, Großbritannien, Kanada, Schweden und USA) beleuchten, wie diese Demokratien militärische Gewaltanwendung gegen Nichtdemokratien rechtfertigen bzw. wie sie ihre Nichtbeteiligung begründen. Die Analyse umfasst den Golfkrieg 1991, den Kosovokrieg 1999 und den Irakkrieg von 2003. Diese drei Kriege stellen keine klaren Fälle von Selbstverteidigung dar und sind daher zumindest für monadische Theorien des Demokratischen Friedens, die eine inhärente Friedlichkeit und Defensivität von Demokratien behaupten (vgl. Benoit 1996; Rummel 1995), besonders erklärungsbedürftig.

Diese drei Kriege wurden vielfach mit Gründen gerechtfertigt, die typisch für Demokratien zu sein scheinen: erstens »Weltordnungskriege«, um rechtsbasierte Prinzipien internationaler Ordnung zu erhalten und durchzusetzen, zweitens »humanitäre Interventionen« für den Schutz fundamentaler Menschenrechte und drittens den gewaltsamen Regimewechsel hin zur Demokratie. Diese Kriege stehen insofern für eine wertgebundene Außenpolitik, die sich im Laufe der 1990er Jahre zu einem »liberalen Interventionismus« verdichtete. Diese »Doktrin« ist insbesondere mit dem Namen des britischen Premierministers Tony Blair verknüpft, der u.a. in einer einflussreichen Rede während des Kosovokrieges den »neuen« Internationalismus beschwor, der nach dem Ende des Ost-West-Konflikts möglich, aber durch verstärkte Interdependenz auch geradezu notwendig geworden sei. Nötig seien nun mehr als jemals zuvor eine intensive internationale Kooperation sowie die mitunter militärische Verteidigung der Werte einer internationalen Gemeinschaft:

»Our armed forces have been busier than ever - delivering humanitarian aid, deterring attacks on defenceless people, backing up UN resolutions and occasionally engaging in major wars as we did in the Gulf in 1991 and are currently doing in the Balkans. [...] Now our actions are guided by a more subtle blend of mutual self interest and moral purpose in defending the values we cherish. In the end values and interests merge. If we can establish

1 Wir danken der Deutschen Forschungsgemeinschaft für die finanzielle Förderung des dem Beitrag zugrunde liegenden Forschungsprojekts über »demokratische Kriege«. Das Projekt wurde im Rahmen des Forschungsprogramms »Antinomien des Demokratischen Friedens « der Hessischen Stiftung Friedens- und Konfliktforschung (HSFK) durchgeführt.

Wir danken Lothar Brock für seinen substantiellen Beitrag zur theoretischen Ausarbeitung des Projekts; für ihre Beiträge zur empirischen Analyse danken wir Una Becker-Jakob, Johanna Eckert, Marco Fey, Stephanie Sohnius, Carmen Wunderlich, Karl Hampel, Susanne Hemmerling und Annabel Schmitz. Für hilfreiche Anregungen und Kritiken vorheriger Fassungen des Artikels danken wir John MacMillan, Dirk Peters, Michael Zürn, den Teilnehmerinnen und Teilnehmern des wissenschaftlichen Kolloquiums der HSFK sowie den anonymen Gutachterinnen und Gutachtern der ZIB. 
and spread the values of liberty, the rule of law, human rights and an open society, then that is in our national interests too. The spread of our values makes us safer« (Blair 1999).

Der vorliegende Beitrag geht solchen Rechtfertigungen liberal-demokratischer Kriegsbeteiligung, aber auch der begründeten Nichtbeteiligung von Demokratien, systematischer nach, indem Aggregatdaten aus einer vergleichenden Analyse parlamentarischer Debatten ${ }^{2}$ vorgestellt werden. Sozialkonstruktivistische Ansätze mit ihrer starken Fokussierung auf die Rolle von Normen erweisen sich hierbei als sehr hilfreich, um die Teilnahme und Nichtteilnahme der untersuchten Länder besser zu erklären. Liberale Normen schränken zwar das Gesamtspektrum rechtfertigbaren Gewaltverhaltens von westlichen Demokratien deutlich ein, jedoch existiert innerhalb dieses Spektrums noch ein relativ großer Interpretationsfreiraum für die Deutung von Normen, der stark durch nationale Identitäten und außenpolitische Rollenkonzeptionen des einzelnen Staates geprägt ist. Dies wiederum ermöglicht verschiedene Verhaltensweisen in der Außenpolitik. »Ideelle« Faktoren erweisen sich so als wichtige, wenn auch selbstredend nicht einzige, Erklärungsfaktoren für die Varianz zwischen den Demokratien.

Im Folgenden wird zunächst der theoretische Ansatz des »demokratischen Krieges« als Antinomie des Demokratischen Friedens skizziert (Abschnitt 2) und anschließend die Methodik erläutert (Abschnitt 3). Die Vorstellung ausgewählter empirischer Resultate der Inhaltsanalyse (Abschnitt 4) ist in der tiefer gehenden Analyse aus Platzgründen auf Untersuchungsergebnisse für die USA, Deutschland, Schweden und Australien beschränkt. Abschließend sind einige Schlussfolgerungen für die Forschung zum »demokratischen Frieden« und »demokratischen Krieg« zu ziehen (Abschnitt 5).

\section{Demokratische Kriege als Antinomien des Demokratischen Friedens}

\subsection{Antinomien des Demokratischen Friedens}

Das Gros der Forschung zum DF stützt sich auf dyadische Varianten der Theorie. ${ }^{3}$ Der einschlägige Doppelbefund besagt, dass Demokratien untereinander keine Kriege führen, aber insgesamt nicht weniger unfriedlich sind als andere politische Systeme (Risse-Kappen 1995). Im letzten Jahrzehnt hat die monadische Theorievariante allerdings Boden gut gemacht: Eine Reihe von Autorinnen und Autoren argumentiert, dass der klassische Kantsche Ansatz zutreffe, der Demokratien insgesamt eine höhere Friedlichkeit zuschreibt (vgl. Czempiel 1996: 79-84; Dieterich et al. 2009: 6-12): Sie

2 Es sei hier betont, dass die Inhaltsanalyse der Parlamentsdebatten nur einen Teil des Forschungsprojekts ausmacht, das auch Rekonstruktionen der nationalen Entscheidungsprozesse, Analysen von Zeitungskommentaren und die Untersuchung von Meinungsumfragen einschließt. Für eine Gesamtdarstellung dieser Ergebnisse siehe Geis et al. (2010).

3 Ein Überblick über die weitgreifende Literatur des Demokratischen Friedens würde den Rahmen dieses Artikels sprengen, siehe dazu Geis (2001), Hasenclever (2003), Müller/Wolff (2006) und Geis/Wagner (2011). 
seien etwas weniger in Kriege und militärische Dispute verwickelt, als es statistisch zu erwarten wäre; sie würden sowohl Kriege als auch militarisierte zwischenstaatliche Dispute seltener initiieren und sich eher um eine friedliche Konfliktlösung bemühen (Russett/Oneal 2001: 95-96, 116, 122; MacMillan 2003). Die Vertreter der monadischen DF-Theorie räumen allerdings auch ein, dass die statistische Evidenz für die allgemeine Friedfertigkeit der Demokratien deutlich schwächer ausgeprägt ist als diejenige für den »demokratischen Separatfrieden«. Auch sie konzedieren, dass Demokratien gelegentlich Kriege gegen Nichtdemokratien ausfechten und auch initiieren - allerdings weniger häufig, als zu erwarten wäre, wenn sich Demokratien und Autokratien nicht unterscheiden würden. Daraus folgt, dass eine kohärente Theorie des Demokratischen Friedens erst dann vollständig wäre, wenn sie den Zusammenhang zwischen Demokratie, Krieg und Frieden systematisch erklären könnte (vgl. Daase 2004: 54).

Die Kriege seit 1990, an denen Demokratien beteiligt waren, lenken nunmehr verstärkt die Aufmerksamkeit auf das »militante« Gesicht von westlichen Demokratien, da der »demokratische Interventionismus« zuzunehmen scheint (vgl. Gleditsch et al. 2007: 11-20). Demokratische Akteure rekurrieren in ihren Rechtfertigungen, wie das Zitat von Tony Blair exemplarisch zeigt, nicht nur offensiv auf liberale Gründe und Motivationen, sondern sie tragen seit Ende des Kalten Krieges insgesamt auch entscheidend zur globalen Transformation des Krieges bei (Chojnacki 2006: 24-26): mittels ihrer Rüstungspolitik (Schörnig 2007; Singer 2009), ihrer Rüstungskontrollund Nichtweiterverbreitungspolitiken (Müller/Becker 2008), ihrer Kriegführung (Shaw 2005) sowie ihrer Bündnispolitiken in Koalitionen und Allianzen. Diese Beobachtungen legen es nahe, das zunächst intuitive Konzept eines »demokratischen Krieges« als das Gegenstück zum Demokratischen Frieden auf seine Stichhaltigkeit hin zu überprüfen.

Der »demokratische Krieg« lässt sich als Antinomie des »demokratischen Friedens« konzeptualisieren (Geis et al. 2006; 2007): Unter »demokratischem Krieg« werden hier demokratie- und/oder liberalismusspezifische Anreize zur Gewaltanwendung, dementsprechende Rechtfertigungen von Militäreinsätzen und spezifische Arten der Kriegführung verstanden (Brock et al. 2006: 208-211). Die Trennung zwischen monadischen und dyadischen Ebenen der DF-Theorien ist weit weniger scharf als gemeinhin suggeriert wird, da die kausalen Annahmen der dyadischen Theorien im Wesentlichen auf denjenigen der monadischen aufbauen, d.h. auf spezifischen Eigenschaften demokratischer Gesellschaften und politischer Systeme, die in der demokratischen Dyade eine überproportionale Wirkung entfalten können (Müller 2002: 48). ${ }^{4}$ Antinomien weisen auf Argumente innerhalb der zahlreichen Varianten der DFTheorien hin, die vernachlässigt oder in ihrer Komplexität nicht reflektiert wurden und zu Widersprüchen innerhalb des Demokratischen Friedens führen können (Müller 2002). Diese verdeckten Wirkungsweisen können überraschende Wirkungen ent-

4 Die rein dyadische rationalistische Theorie von Bueno de Mesquita et al. (1999) über »demokratische Abschreckung « mag als Ausnahme gelten, die zwar mit den Aggregatdaten der Statistik, aber nicht mit den Befunden der einschlägigen Fallstudien übereinstimmt (Müller/ Wolff 2006: 55-58). 
falten, die durch die Hypothesen des theoretischen Mainstreams nicht abgedeckt sind. Im Bereich Krieg/Frieden lassen sich drei zentrale Antinomien aus der DF-Theorie ableiten:

(1) Der utilitaristische Kausalmechanismus der DF-Theorie beruht auf der Annahme, dass Bürgerinnen und Bürger in der Regel die Kosten eines Krieges scheuen und gewählte Regierungen sich aus diesem Grund kriegsabgeneigt zeigen (Bueno de Mesquita et al. 1999). Allerdings können sich Kosten-Nutzen-Kalküle unter bestimmten Bedingungen verändern und sich daraus auch positive Erwartungen bezüglich eines die Kosten überwiegenden Nutzens eines Krieges ergeben. Gerade seit Ende des Kalten Krieges sind international verschiedene Tendenzen zu verzeichnen, welche aus Sicht politischer und militärischer Führungen die politischen Risiken und menschlichen wie materiellen Kosten der Kriegführung von Demokratien zu senken versprechen - etwa durch eine Hightech-Rüstungspolitik der NATO-Staaten, die sog. Revolution in Military Affairs (O'Hanlon 2000; Schörnig 2007), oder den verstärkten Einsatz privater Sicherheitsunternehmen (Deitelhoff 2009). Solche Entwicklungen können dazu beitragen, einen zentralen »Kostenfaktor« in liberal-demokratischen Gesellschaften abzumildern: die Angst vor menschlichen Opfern, speziell unter den eigenen Soldaten (Mandel 2004: 11; Shaw 2005: 79-83). Die sog. Opfersensibilität westlicher Demokratien ist in den letzten Jahrzehnten eher noch gewachsen, da das einzelne Individuum immer besser »versichert « wird und das Leben des/der Einzelnen eine noch größere Wertschätzung genießt. Viele westliche Gesellschaften gelten daher bereits als »post-heroische« Gesellschaften, worauf die politischen und militärischen Eliten in ihren Strategien der Kriegführung Rücksicht nehmen müssten (Shaw 2005).

Kosten-Nutzen-Kalküle der Öffentlichkeit können darüber hinaus auch durch stark asymmetrische Machtbeziehungen zwischen dem eigenen Staat (und seinen Verbündeten) und dem gegnerischen Staat zugunsten von Gewaltanwendung beeinflusst werden. Es ist auffällig, dass die größeren Interventionen und Kriege der Demokratien seit den 1990er Jahren durch solche asymmetrischen Machtverhältnisse gekennzeichnet waren und keine Demokratie ohne Verbündete militärisch aktiv wurde. Durch ad-hoc-Bündnisse oder durch Allianzen wie die NATO bündeln Demokratien ihre militärischen und ökonomischen Ressourcen, wodurch die Risiken einer Militärintervention für den einzelnen Teilnehmer reduziert werden können (Chojnacki 2006: 29). Andererseits werden Kriege nicht einfach deswegen geführt, weil ihre Kosten den Demokratien erträglich erscheinen. Hier spielen weitere Faktoren eine Rolle - das Kosten-Nutzen-Kalkül wirkt somit nicht als unabhängige Variable.

(2) Die normativ-kulturelle Erklärung des Demokratischen Friedens nimmt an, dass demokratische Bürger einer konsolidierten Demokratie liberal-demokratische Werte und Normen verinnerlicht hätten, welche die Achtung vor dem Mitbürger und friedliche Formen der Konfliktlösung befördern. Demokratische Politiker strebten danach, die im Innern der Demokratien praktizierten Formen des Ausgleichs und des fairen Umgangs miteinander auch nach außen zu übertragen (Risse-Kappen 1995: 501; Dixon/Senese 2002). Allerdings können demokratische Akteure gerade mit dem Bezug auf liberal-demokratische Werte Militäreinsätze erwägen und rechtfertigen. Wenn die 
Verteidigung von Menschenrechten, die Verhinderung von Genozid oder die Verbreitung von Demokratie Bestandteile der Identitäts- bzw. außenpolitischen Rollenkonzeption eines demokratischen Staates sind, kann dies seine Interventionsneigung erhöhen.

Normative Einstellungen zum Einsatz von Gewalt sind geprägt von historischen Erfahrungen und daher in der kollektiven Identität bzw. der politischen Kultur einer Gesellschaft verankert (Katzenstein 1996; Duffield 1999; Schwab-Trapp 2002). Konstruktivistische Ansätze der Außenpolitikanalyse basieren auf der Annahme, dass außenpolitische Entscheidungen von solchen normativen Einstellungen beeinflusst werden (Boekle et al. 2001; Stahl 2006). Die Einstellung einer Bevölkerung bezüglich der Legitimität militärischer Gewaltanwendung ist überdies abhängig von der Rollenkonzeption, die »ihr« Staat in der Interaktion mit wichtigen außenpolitischen Partnern für sich entwickelt und in der eigenen Außenpolitik anwendet. Diese Rollenkonzepte bestehen aus dem (wandelbaren) Selbstbild, das aus der nationalen Identität und politischen Kultur entspringt, und den (wandelbaren) normativen Erwartungen anderer Staaten, zu denen sich der »eigene« Staat verhält (Kirste/Maull 1996; Harnisch/Maull 2001; Stahl 2006; Müller 2007). Da die Konstruktion und Reproduktion kollektiver Identitäten von der Differenzierung zwischen einem »Ich/Wir« von einem »Anderen/Sie« abhängt, können solche Abgrenzungsprozesse zur Entstehung von Feindbildern führen (Kennedy 1997). Wird ein Gegner als bösartiger Feind etikettiert, der all die geschätzten westlichen Werte verneint und bekämpft, so kann dies liberale Militanz erhöhen und die »ingroup/outgroup«-Dynamik zwischen »guten« Demokratien und »bösen« Diktaturen verschärfen (vgl. Risse-Kappen 1995: 506-507).

(3) Viele Erklärungen des DF fußen auf institutionalistischen Argumentationen, die zumeist die verfassungsmäßigen Institutionen der Demokratien als komplexe, kriegsbehindernde Konstellationen von institutionellen Vetospielern behandeln (Auerswald 1999; Dieterich et al. 2009). Aus der Vielzahl vermuteter institutionalistischer Kausalmechanismen richtet sich die vorliegende Untersuchung indessen auf zwei, die auf der internationalen Ebene angesiedelt sind und häufig von der englischsprachigen DF-Forschung vernachlässigt werden: internationale Organisationen und Völkerrecht. Demnach zeigen Demokratien eine besondere Neigung, internationalen Organisationen beizutreten, die wiederum Kompromisse und friedliche Konfliktlösungen finden helfen (Russett/Oneal 2001: 157-196; Dembinski et al. 2009: 7-11). Diese Organisationsneigung kann jedoch auch eine Kehrseite aufweisen, insofern Mitgliedschaft in einer internationalen Organisation Demonstrationen von Loyalität und das Einverständnis mit kollektiven Entscheidungen implizieren kann, die der Staat für sich allein nicht getroffen hätte. Institutionszugehörigkeit verlangt regelbasiertes Verhalten, d.h., sie schafft eine eigene Angemessenheitslogik, welche die Handlungsfreiheit beschränken kann. Wenn Demokratien in besonderer Weise durch gemeinsam geschaffene Normen und Regeln gebunden sind, so ist zu erwarten, dass sie ausgesprochen loyale Mitglieder ihrer Bündnisse sind (Lipson 2003). 
Was die Rolle des Völkerrechts in der englischsprachigen DF-Forschung anbelangt, so ist diese bislang seltsam blass geblieben. ${ }^{5}$ Dies ist insofern schwer nachvollziehbar, als Völkerrecht liberale Außenpolitik, insbesondere Entscheidungen über Krieg und Frieden, wesentlich strukturiert (Liste 2009: Kap. 1-3). Die argumentativen Referenzmuster in innerstaatlichen Debatten über Kriegsentscheidungen sind daher in der Regel eng mit der internationalen Normstruktur verknüpft, die ihrerseits stark von Demokratien geprägt wird (vgl. Wiener 2004). Jedoch lassen sich aus der völkerrechtlichen Strukturierung der Kriegsentscheidungen keine eindeutigen Erwartungen über das Verhalten einzelner Demokratien ableiten, da die Auslegung völkerrechtlicher Normen zum einen umstritten ist, zum anderen westliche Demokratien zum Schutz ihrer Volkssouveränität und nationalstaatlichen Autonomie eine allzu enge Bindung an die prozeduralen Normen des Völkerrechts scheuen (Brock 2007: 66). Allerdings würde auch eine sehr enge Bindung an das (Völker-)Recht nicht zwingend zur Friedlichkeit von Demokratien führen, da sich seit 1990 ein internationaler Normwandel vollzieht, in dem die Staatenpraxis westlicher Demokratien eine entscheidende Rolle spielt und der die Interventionstätigkeit von Demokratien befördert hat. Infolge u.a. von »humanitären Interventionen« und einer Militarisierung der Terrorismusbekämpfung ist eine Schwächung grundlegender Normen der internationalen Gesellschaft zu beobachten, in der sich Staaten wechselseitig als Gleiche und Souveräne anerkennen und sich daher der Intervention enthalten (Zangl/Zürn 2003: 218-268; Brock 2007: 53-60).

Die drei zentralen Antinomien im Themenfeld Krieg/Frieden verweisen so insgesamt auf eine demokratieimmanente Neutralisierung der vermuteten Friedfertigkeit der Demokratien und/oder sogar auf eine demokratietypische Kriegsneigung: Wie können die institutionellen und kulturell-normativen Kriegshemmnisse, die innerhalb von Demokratien wirksam sein sollen (Russett 1993: 30-42), im Falle von Kriegsbeteiligungen überwunden werden? Warum werden sie im Falle der Nichtteilnahme gestützt oder verstärkt? Gibt es womöglich demokratiespezifische bzw. dem Liberalismus inhärente Gründe, Kriegshemmnisse außer Kraft zu setzen? In der klassischen DF-Forschung ordneten sich zahlreiche Erklärungsansätze lange Zeit entlang einer Scheidelinie von rein institutionalistischen Ansätzen und stärker normativen sowie sozialkonstruktivistischen Argumentationen. ${ }^{6}$ Obwohl Normen und Institutionen nicht zu trennen sind, solche Ansätze also kombiniert werden sollten, klammert die folgende Analyse die Rolle von innerdemokratischen Institutionen ${ }^{7}$ aus Platzgründen aus und beschränkt sich insofern auf eine Weiterführung normativ-kultureller Ansätze des Demokratischen Friedens.

5 In der deutschsprachigen DF-Literatur ist diese völkerrechtliche Leerstelle dagegen problematisiert worden; für konstruktive Vorschläge, wie Problematiken des Demokratischen Friedens mit völkerrechtlichen Argumentationen verknüpft werden können, siehe etwa Brock (2007), Eberl/Fischer-Lescano (2005) und Liste (2009).

6 Ausführlich dazu Geis (2001), Müller/Wolff (2006) und Geis/Wagner (2011).

7 Siehe hierzu jüngst etwa die Untersuchung zum »parlamentarisch-demokratischen Frieden« von Dieterich et al. (2009) sowie Peters/Wagner (2009). 


\subsection{Die Legitimität des Krieges in liberalen Demokratien: Eine liberal- konstruktivistische Sicht auf Präferenzen und Interessen}

Da die hier vorgestellten Ergebnisse einer vergleichenden Inhaltsanalyse einen Beitrag zur sozialkonstruktivistischen Forschung darstellen, beruhen sie auf »weicheren« Vorstellungen von Kausalität. »Norms are causal insofar as they regulate behaviour. Reasons are causes to the extent that they provide motivation and energy for action" (Wendt 1999: 82). Normen strukturieren politische Entscheidungen, sie ermöglichen und beschränken zugleich die angemessenen Handlungsoptionen. Daher ist es sehr wichtig - und nicht »bloß« Rhetorik -, welche öffentlichen Rechtfertigungen politische Eliten benutzen, um ihre Entscheidungen zu legitimieren, und welche Gründe als legitim akzeptiert werden von »ihrer« Öffentlichkeit. Außenpolitische Interessen der Demokratien sind daher nicht fixiert oder durch einen systemischen Zwang oktroyiert (Kahl 1999: 107-108). Was genau im Interesse eines gegebenen demokratischen Staates in einer konkreten Situation liegt, ist gemeinhin innenpolitisch umstritten, ist Gegenstand öffentlicher Debatten und politischer Entscheidungsprozesse, wobei die Positionen in diesen Debatten wiederum pfadabhängig historisch gewachsen sind (Czempiel 1996: 83-84; Hellmann 2006: Kap. 9-12).

In der heutigen Welt sind die Interessen und Präferenzen westlicher Demokratien durch eine hohe Interdependenz beeinflusst, durch Meinungsverschiedenheiten über die »richtige« Interpretation des Völkerrechts und die angemessene Beziehung zum »Anderen«, also zu den Nichtdemokratien im internationalen System. Die Auflösung der Bipolarität hat in der Phase nach dem Kalten Krieg eine allgemeine Unsicherheit über den »richtigen « Stil, die Ausrichtung und den Inhalt liberal-demokratischer AuBenpolitik erzeugt (Freedman 2005: 94). Es ist unklar, was das langfristige Ziel einer »liberalen Weltordnung« eigentlich beinhaltet. Schließlich umfassen Interessen demokratischer Staaten nicht nur Machtinteressen im klassischen realistischen Verständnis, sondern gleichfalls die Verwirklichung "weicher« normativer Ziele, z.B. der weltweiten Verbreitung von Menschenrechten und Demokratie (vgl. Chafetz et al. 1999: xvi; Spanger/Wolff 2007: 263-273).

Daraus folgt, dass detailliert und differenziert zu ermitteln ist, welche Art von Interessen, Präferenzen und wertgebundenen Zielen in konkreten Situationen politischer Entscheidung artikuliert werden. Dem Beschluss, ob sich eine Demokratie an einer Intervention oder einem Krieg beteiligt, gehen zumeist öffentliche Debatten und intensive Entscheidungsprozesse voraus. Die Friedfertigkeit oder Kriegsbereitschaft einer Demokratie hängt somit entscheidend von den Mehrheitspräferenzen innerhalb der Bevölkerung und der politischen Akteure ab, die in der öffentlichen Debatte in einem erheblichen Maß geformt und modifiziert werden können. Die Forschung zum Demokratischen Frieden behandelt solche Präferenzen überwiegend als exogen gegeben und nimmt an, dass Bürgerinnen und Bürger grundsätzlich friedliche Präferenzen haben, während Regierungen Sonderinteressen an der Kriegsbeteiligung hegen können und des beschränkenden Einflusses des Volkes bedürfen, um von militärischen Einsätzen abgehalten zu werden (vgl. Kahl 1999: 112). Tatsächlich jedoch können Bürgerinnen und Bürger alle möglichen Einstellungen zu Militäreinsätzen 
aufweisen. Diese Einstellungen variieren mit dem Typ des Einsatzes, um den es geht. Von enthusiastischer Unterstützung über rationale Billigung und Indifferenz bis zu leidenschaftlicher Gegnerschaft reicht die Palette (vgl. Reiter/Stam 2002: 144-163). In den sieben Länderstudien des diesem Beitrag zugrunde liegenden Forschungsprojekts zeigte sich, dass zumindest laut nationaler Meinungsumfragen im Vorfeld der Kriege die Regierungen bei insgesamt zehn von 13 Entscheidungen für eine Kriegsbeteiligung im Einklang mit der Mehrheitsmeinung ihrer Öffentlichkeiten handelten. ${ }^{8}$

Vor diesem Hintergrund ist es weiterführend, mit Hilfe von Inhaltsanalysen die Legitimations- und Rechtfertigungsmuster zu identifizieren, mit denen politische Eliten den Einsatz von militärischer Gewalt im Rahmen von Parlamentsdebatten öffentlich begründen oder ablehnen. Kritikerinnen und Kritiker von Inhaltsanalysen monieren, dass diese nicht die »tatsächlichen « Gründe ermitteln können, welche die individuellen Akteure zu ihren Kriegs- oder Antikriegsentscheidungen bewogen haben, sondern lediglich die Intensität der Lippenbekenntnisse gegenüber der Öffentlichkeit messen (vgl. Mearsheimer 2001: 25). Zwar ist es in der Tat nicht möglich, durch Inhaltsanalysen die individuellen Motivationen der Sprecher zweifelsfrei festzustellen, da Akteure strategisch handeln können, wenn sie sich über ihre Motivation für eine bestimmte Handlung oder ihre Position zu einer Streitfrage äußern. Dennoch ist die Inhaltsanalyse von Debatten aus sozialkonstruktivistischer Perspektive sehr aufschlussreich:

Erstens müssen Akteure, die öffentlich ein Argument vorbringen - sei dies nun in strategischer Absicht oder »aufrichtig « - damit rechnen, dass andere Sprecherinnen und Sprecher (»Empfänger«) dieses ernst nehmen und die »Sender« in der öffentlichen Debatte dafür in Haftung nehmen. Die »Sender« sind um ihrer Glaubwürdigkeit willen gezwungen, sich so zu verhalten, als ob sie die Argumente aufrichtig vertreten hätten (»argumentative self-entrapment«, Risse 2000: 32; Schimmelfennig 2001: 65-66). Zweitens ist es wahrscheinlich, dass demokratisch gewählte Akteure, die ihre Absichten oder Handlungen öffentlich rechtfertigen müssen, um Zustimmung zu erlangen, »resonanzkräftige« Argumente vorbringen; d.h. solche, von denen sie glauben, dass sie den Wertorientierungen und Interessen ihrer Zuhörerschaft am ehesten entsprechen. Dies gilt im Besonderen für parlamentarische Debatten, in denen die Sprecherinnen und Sprecher ihre Positionen gegen die Opposition und vor der Öffentlichkeit zu verteidigen haben. ${ }^{9}$

Bei einer Inhaltsanalyse öffentlicher Diskurse zählen daher nicht die individuellen Motive, sondern sozial geteilte, öffentlich »hergestellte« Interpretationen (Baumann

8 Die drei Ausnahmen waren: die kanadische Regierung unter Premierminister Mulroney für den Golfkrieg von 1991, die britische Regierung unter Premierminister Blair sowie diejenige Australiens unter Premierminister Howard für den Irakkrieg von 2003.

9 Da es im vorliegenden Beitrag um die Identifizierung der Rechtfertigungsmuster für und gegen einen Kriegseinsatz geht, ist im Übrigen die Parteizugehörigkeit der jeweiligen Sprecherinnen und Sprecher von sekundärer Bedeutung. Entscheidend ist vielmehr die Unterscheidung zwischen »Kriegsbefürworterinnen und Kriegsbefürwortern« und »Kriegsgegnerinnen und Kriegsgegnern« - eine Dichotomie, die mit der Differenz von Regierungspartei(en) und Oppositionspartei(en) identisch sein kann, aber nicht identisch sein muss. 
2006: 98). Es ist unerheblich, ob Argumente nur »vorgeschoben« sind, weil für die vorliegende Fragestellung entscheidend ist, dass der Austausch bestimmter Argumente von einer »politischen Praxis begleitet wird, die diese Argumente in ihrem Handeln als Gründe für ihre Entscheidungen ausweist« (Schwab-Trapp 2002: 47). Da demokratische Regierungen und Abgeordnete die mehrheitliche Zustimmung von Parlament und Öffentlichkeit für ihre Positionen anstreben, lässt sich durch die Inhaltsanalyse parlamentarischer Reden in vergleichender Absicht am systematischsten rekonstruieren, auf Basis welcher Rechtfertigungen eine bestimmte Demokratie in den Krieg zieht (oder sich dagegen entscheidet). ${ }^{10}$

\section{Methodik: Kodierverfahren, Kategorienschema und Daten}

Die in Abschnitt 4 folgenden Ergebnisse der Inhaltsanalyse beruhen auf der Auswertung von 781 parlamentarischen Reden ${ }^{11}$ in Australien, Deutschland, Frankreich, Großbritannien, Kanada, Schweden und den USA, die für oder gegen eine eigene Kriegsbeteiligung ihres Landes - hier verstanden als die Entsendung eigener bewaffneter Truppen ${ }^{12}$ - im Vorfeld des Golfkriegs 1991, des Kosovokriegs 1999 und des Irakkriegs 2003 argumentierten. ${ }^{13}$ Die Fallauswahl der sieben Staaten beschränkte sich auf konsolidierte westliche Demokratien, um so auf der Seite der unabhängigen Variablen vergleichsweise ähnliche Fälle zu erhalten. Gleichwohl wurden bei der Fallauswahl auch bestimmte demokratieunabhängige Einflussfaktoren berücksichtigt, die auf die rationalistischen Kosten-Nutzen-Kalküle demokratischer Akteure oder deren normative Einstellungen zu Militäreinsätzen einwirken und so die Friedlichkeit von Demokratien außer Kraft setzen können: So wurden Demokratien un-

10 Natürlich schließt dieser Ansatz nicht aus, dass sich ein einzelner Sprecher oder eine Sprecherin über die Interessen und Wertorientierungen der Zuhörerschaft und die darauf beruhenden erwünschten Rechtfertigungsmuster täuscht oder Sonderinteressen anspricht, die nicht die Präferenzen der Gesellschaft als Ganzes repräsentieren. Indessen sollte dieses Problem vernachlässigbar sein, wenn die Zahl der Sprecherinnen und Sprecher groß genug ist.

11 Diese schließen in den parlamentarischen Demokratien Regierungsmitglieder ein. In den (semi-)präsidentiellen Systemen der USA und Frankreichs, in denen der Präsident nicht vor dem Parlament spricht, wurden zusätzlich zu den Parlamentsreden eine Zufallsauswahl von öffentlichen Reden der Exekutive in die Grundgesamtheit aufgenommen. Die Entscheidung für Parlamentsdebatten hatte einen praktischen und einen theoretischen Grund: Der praktische Grund lag in der leichten Zugänglichkeit relevanter Daten, der theoretische ist in der Rolle von Parlamenten in Demokratien verwurzelt. Sie sind Arenen, in denen die Vertreterinnen und Vertreter der politischen Eliten ihre Positionen zu politisch wichtigen Fragen vor einer (medial vermittelten) Öffentlichkeit präsentieren. Parlamentsdebatten bieten daher einen idealen Untersuchungsraum, um an die Öffentlichkeit gerichtete Rechtfertigungsmuster zu ermitteln. Davon unbenommen ist die nicht vernachlässigbare Rolle anderer Teile der Öffentlichkeit - Medien, Parteien, Nichtregierungsorganisationen, Kirchen - im öffentlichen Diskurs.

12 Für eine differenziertere Typologie von »Kriegsbeteiligung« siehe Dieterich et al. (2009: 22-25).

13 Der Datensatz ist als SPSS-Datei online einzusehen unter http://hsfk.de/Ursachen-derwechselnden-Beteiligung-demokratisch.140.0.html. Dort findet sich auch das Kodierbuch. 
terschiedlicher Statuskategorien einbezogen (Supermacht, Großmacht, Mittelmacht), alliierte und nicht-alliierte Demokratien sowie, im Hinblick auf kollektive Identitäten und außenpolitische Rollen(selbst)zuschreibungen, Demokratien auf dem breiten Spektrum zwischen den idealtypischen Polen Militärmacht und Zivilmacht bzw. einer »militanten« und »pazifistischen« politischen Kultur (Müller 2004; 2007). Dabei sollte jedoch kein klassischer Theorientest erfolgen, sondern der Einfluss dieser Faktoren in den Entscheidungs- und Legitimationsprozessen im Vorfeld der Kriege untersucht werden.

Die drei ausgewählten Kriege waren aus Sicht der Demokratien »wars of choice « (vgl. Freedman 2006/7: 52), d.h. sie stellten keine klaren Fälle von Selbstverteidigung dar ${ }^{14}$ und sind daher zumindest für monadische Theorien des Demokratischen Friedens besonders erklärungsbedürftig. Dies trifft auch auf den Irakkrieg von 2003 zu, der - insofern er denn mit der nationalen Sicherheit der Kriegführenden verbunden wurde - immer noch mit dem Zweck der Prävention gerechtfertigt wurde, die nach herrschender Meinung nicht unter die Kategorie der Verteidigungskriege subsumiert werden kann. Die drei Kriege wurden zudem von keiner Demokratie allein ausgetragen, sondern jeweils im Verbund mit anderen (ob NATO oder »coalitions of the willing «).

Die Inhaltsanalyse diente einem doppelten Ziel: Erstens sollten die wichtigen Argumente identifiziert werden, die darauf abstellen, den vermuteten Widerstand gegen eine Kriegsbeteiligung zu überwinden oder zu bestärken. Mit anderen Worten: Mit welchen Argumenten legitimieren politische Eliten gegenüber der Wählerschaft diese Entscheidungen? Das zweite Ziel ist mit dem ersten verknüpft, geht jedoch weiter: Gibt es besondere Argumente für oder gegen Krieg, die von der anderen Seite als relevant und legitim angesehen werden, die aber durch Gegenargumente in einem Abwägungsprozess außer Kraft gesetzt werden können? Setzen sich die Sprecherinnen und Sprecher also mit spezifischen Argumenten der Gegenseite auseinander, womit sie zusätzliche Hinweise auf deren Relevanz für die Bevölkerung geben? Der Blick auf diesen Abwägungsprozess bietet Aufschlüsse über die demokratischen Kriegshemmnisse sowie die Argumentationsstrategien, um diese zu überwinden.

Für jedes Land und jeden der drei Konflikte wurde die Inhaltsanalyse über denselben Untersuchungszeitraum durchgeführt, der mit dem Tag des Kriegsbeginns endete, um einen rally around the flag-Effekt auszuschließen. ${ }^{15}$ Analyseeinheit bildete die einzelne Rede. Es durften nur Reden kodiert werden, in denen der Sprecher/die Sprecherin eine klare Position zur Entsendung eigener bewaffneter Truppen einnahm. Dies ergab sich aus der Zielsetzung der Untersuchung, diejenigen Argumente zu erheben, die von Befürworterinnen und Befürwortern und Gegnerinnen und Gegnern

14 Aus diesem Grund wurde hier nicht der Afghanistan-Krieg einbezogen: Aus Sicht der USA stellte dieser nach den Terroranschlägen vom 11. September 2001 eine legitime Form der Selbstverteidigung dar; diese Sicht machten sich auch ihre NATO-Verbündeten zu eigen, die den Bündnisfall ausriefen. Die vorliegende Untersuchung beurteilt nicht, ob solche Deutungen und Rechtfertigungen angemessen sind oder nicht, sondern erhebt, welche Rechtfertigungen die öffentlichen Diskurse einer Demokratie jeweils dominieren.

15 Die genauen Untersuchungszeiträume waren: Golfkrieg 2.8.1990-17.1.1991; Kosovokrieg 23.9.1998-24.3.1999; Irakkrieg 8.7.2002-20.3.2003. 
einer eigenen Truppenbeteiligung jeweils als überzeugend wahrgenommen wurden. Reden, in denen sich der Sprecher/die Sprecherin nicht festlegte oder die sich ausschließlich auf die Entsendung fremder Truppen bezogen, blieben unberücksichtigt. Diese Truppenentsendung konnte entweder erst dem Aufbau einer »Drohkulisse« nahe dem Konfliktgebiet dienen oder bereits explizit mit dem möglichen Einsatz dieser Truppen verknüpft sein.

Es sollten pro Land und Konflikt 50 zufällig gezogene Reden kodiert werden. Erste Materialsichtungen hatten gezeigt, dass dies die größte realistisch zu erwartende kodierbare Zahl von Reden für alle Länder darzustellen schien. Um die Samples ungefähr gleich groß zu halten, wurde die Anzahl der Reden entsprechend nach oben hin begrenzt. Allerdings stellte sich heraus, dass es in einigen Ländern und Konflikten nicht möglich war, jeweils 50 Reden zu identifizieren, die den Kodieranforderungen genügten. Dies lag an der wahrgenommenen geringeren direkten Betroffenheit des eigenen Landes, an spezifischen Debattierkulturen, an der nur sehr begrenzten Mitwirkung von Parlamenten an Kriegsentscheidungen (vgl. Dieterich et al. 2009) oder am Unwillen einiger Abgeordneten, sich auf eine klare Position festzulegen. In diesen Fällen wurde eine Vollerhebung aller kodierbaren Reden durchgeführt.

Die Reden wurden mithilfe der Software Atlas-ti kodiert, auf Unterstützung durch vorgegebene Schlüsselbegriffe wurde verzichtet (vgl. Kantner et al. 2008: 6-9). Alle Argumente wurden binär als Dummyvariablen kodiert, d.h. als in der Rede entweder vorkommend oder nicht erwähnt. ${ }^{16}$ Um die Reliabilität der Kodierung zu vergrößern, wurden alle Texte getrennt von zwei, in Streitfällen drei, Personen kodiert und anschließend diskutiert. Die Auswertung der Daten erfolgte in SPSS.

Grundlage der Inhaltsanalyse war ein Kategorienschema von 45 Argumenten (siehe das Kategorienschema im Anhang 1). Je eine Gruppe von Argumenten waren prooder contra-Argumente hinsichtlich einer Teilnahme an militärischen Operationen; eine gleichsam »mittlere«, den pro-Argumenten zugerechnete, Kategorie bildeten Argumente, die eine Entscheidung pro Kriegsbeteiligung »begünstigen«, jedoch für sich allein genommen nicht rechtfertigen konnten, z.B. der Verweis auf die eigene militärische Überlegenheit. Das Kategorienschema entstand zweistufig: Erstens wurden Argumente aus realistischen, institutionalistischen, liberalen und konstruktivistischen IB-Theorien bzw. -Ansätzen sowie aus den in Abschnitt 2.1 diskutierten Antinomien des Demokratischen Friedens deduziert. Zweitens wurden diese durch im Pretest induktiv aufgefundene Argumente ergänzt. ${ }^{17}$ Die 45 Argumente wurden sieben »Frames « zugeordnet, die ihre zentrale Logik bzw. ihren argumentativen Bezug wiedergeben und sich auf Macht, Allianzzugehörigkeit, die nationale Identität/außenpolitische Rolle, das Völkerrecht, universelle Werte, spezifisch demokratische Werte oder eine Feindbildkonstruktion beziehen. Die Frames zeigen also an, wie

16 Das Reliabilitätsniveau aller individuellen Kodiererinnen und Kodierer im Pretest erreichte 0.64 , was angesichts des sehr umfangreichen Kategorienschemas ein akzeptabler Wert ist.

17 Die Beschreibung aller Argumente sowie weitere Hintergrundinformationen zum Kodierprozess können im Kodierbuch nachgelesen werden, das online unter http://hsfk.de/Ursachen-der-wechselnden-Beteiligung-demokratisch.140.0.html verfügbar ist. 
wichtig den Sprecherinnen und Sprechern bestimmte grundsätzliche Argumentationslogiken waren, die sie in den nationalen Parlamentsdebatten für besonders bedeutend oder überzeugend hielten.

Allerdings zeigte sich, dass zwei Argumentationstypen - der Verweis auf eine durch eine Militärentsendung aufzubauende Drohkulisse sowie der Verweis auf ausgeschöpfte bzw. noch nicht ausgeschöpfte friedliche Mittel - trotz einer grundsätzlichen Einordnung in den Macht- bzw. Völkerrechtsframe separat ausgewiesen werden sollten. Beide werfen Probleme und Fragen auf, die über die grundsätzliche Logik des Frames hinausgehen und später noch im Detail besprochen werden. Insgesamt ergeben sich also $7+2$ Frames, die zur Betrachtung der einzelnen Länder herangezogen werden.

Die ausgewerteten Parlamentsreden ermöglichen zwei Arten von Analyse: erstens auf dem Makrolevel die Analyse der Grundgesamtheit aller verfügbaren Reden über alle Länder und Konflikte hinweg. Diese Makrobefunde können natürlich von Land zu Land und von Krieg zu Krieg variieren, aber sie markieren einen interessanten Ausgangspunkt für weitere Untersuchungen. Diese Befunde werden in Abschnitt 4.1 präsentiert. Zweitens ermöglichen die Daten, einzelne Länder und einzelne Konflikte zu betrachten, sowie jede Vorkriegsdebatte in den einzelnen Ländern zu untersuchen und zu vergleichen, um die Verhaltensvarianz zu verstehen. Die in Abschnitt 4.2 folgende Analyse beschränkt sich auf die Betrachtung der einzelnen Länder über alle Konflikte hinweg. Die Darstellung kann hier nicht die Detailauflösung auf Ebene der 45 Argumente abbilden, da die vergleichende Betrachtung mehrerer Länder zu komplex und unübersichtlich würde, sondern zeigt die Ebene der $7+2$ Frames, in denen Argumente mit analoger Argumentationslogik zusammengefasst werden. Entsprechend wird auch nicht zwischen Befürwortung oder Ablehnung des Militäreinsatzes unterschieden, da die argumentative Grundlogik im Fokus steht, die in den Reden als relevant erachtet wird. Trotzdem können so nationale Besonderheiten identifiziert und nationale Argumentationsstrukturen miteinander verglichen werden.

Die Berechnung der Frames erfolgte auf Basis der einzelnen Konflikte, wobei zur besseren Vergleichbarkeit zwischen den Ländern die Stärke der Nutzung eines Arguments mit der durchschnittlichen Häufigkeit aller Argumente im spezifischen Land und Konflikt standardisiert wurde, um seine Relevanz im jeweiligen parlamentarischen Diskurs zu bestimmen - so kann z.B. einem Argument, das in zwei Ländern während eines bestimmten Konflikts von jeweils 30\% der Sprecherinnen und Sprecher genannt wird, aufgrund unterschiedlicher Diskussionskulturen eine unterschiedliche Bedeutung bzw. Relevanz im nationalen Diskurs zukommen. Die einzelnen Ergebnisse wurden für jedes Argument danach zu einem nationalen Gesamtbild über alle drei Konflikte aggregiert und ein Durchschnittswert ermittelt. Diese wurden dann 
gemäß des zugrunde liegenden Arguments den Frames additiv zugeordnet. ${ }^{18}$ Diese Summe gibt dann die absolute Relevanz eines Frames (also einer bestimmten Argumentationslogik) im Rahmen eines nationalen Diskurses an und ermöglicht die Erstellung einzelstaatlicher »Argumentationskarten« (siehe Abschnitt 4.2), welche die Struktur der nationalen parlamentarischen Kriegsdiskurse charakterisieren.

\section{Ausgewählte empirische Ergebnisse}

Dieser Abschnitt gliedert sich in zwei Teile: Zunächst werden auf der Makroebene aller Reden über alle Konflikte und Länder hinweg einige Auffälligkeiten in der allgemeinen Struktur der Debatte über Frieden und Krieg erläutert (Abschnitt 4.1). Zweitens werden Daten der einzelnen Länder vorgestellt, um Aufschlüsse über die Verhaltensunterschiede zwischen den Demokratien geben zu können. Die Detailanalyse in Abschnitt 4.2 muss sich allerdings aus Platzgründen auf die Vorstellung von Ergebnissen zu vier Ländern - USA, Australien, Deutschland, Schweden - beschränken. Diese vier Staaten eignen sich für die Auswahl, da sie hinreichend Varianz in ihrem Verhalten zeigen: Die USA nahmen an allen drei Kriegen teil, Australien an beiden Kriegen am Golf, Deutschland nur am Kosovokrieg und Schweden entsandte in keinem Fall Kampftruppen (lediglich ein Feldhospital im Golfkrieg 1991).

\subsection{Der Weg in den Krieg}

\section{Auseinandersetzung mit den Gegenargumenten in den Parlamenten}

Die meisten demokratischen Parlamente führen Debatten über ihre Militäreinsätze im Vorfeld der Regierungsentscheidungen ${ }^{19}$; ihre verfassungsrechtlich garantierten Kontrollmöglichkeiten variieren jedoch enorm und ihre Abstimmungen - so es sie

18 Gegeben $\mathrm{A}_{\mathrm{ikl}}(\%$ der Parlamentarierinnen und Parlamentarier, die während Konflikt $k$ in Land $l$ das Argument $i$ gebrauchen), erfolgte die Berechnung der »Relevanzen « (also die Standardisierung) wie folgt: $R E L_{i k l}=\frac{A_{i k l}}{\left(\sum_{i=1}^{45} A_{i k l}\right) / 45}$. Die durchschnittliche Relevanz eines Arguments für ein Land über die drei (im Fall Australien: zwei) Konflikte ergibt sich als: $R E L A V_{i l}=\left(\sum_{k=1}^{3} R E L_{i k l}\right) / 3$. Der absolute Wert eines Frames ist die Summe aller Relevanzen der im Frame enthaltenen Argumente. Da die einzelnen Frames jedoch unterschiedlich viele Argumente umfassen (siehe Kategorienschema in Anhang 1), können die einzelnen Frames unterschiedliche (theoretische) Maximalwerte erreichen, was beim direkten Vergleich innerhalb eines Landes berücksichtigt werden muss.

19 Angesichts unseres Untersuchungszeitraums, der mit dem Tag des jeweiligen Kriegsbeginns endete, fand aber z.B. keine Parlamentsdebatte in der französischen Assemblée nationale vor Beginn des Kosovokrieges statt. Das französische Parlament debattierte erst, nachdem der Krieg schon begonnen hatte. Vgl. Stahl (2006: 191-240). 
überhaupt gibt - sind teils symbolischer Natur (Wagner 2006; Dieterich et al. 2009). Die hier erhobenen Daten zeigen, dass die meisten Debatten kontrovers geführt wurden, die Mehrheit der Sprecher allerdings lediglich den eigenen Standpunkt vorträgt und auf die Gegenposition kaum eingeht. Nur 41,7\% aller kriegsbefürwortenden Sprecherinnen und Sprecher und nur 38,1\% der Gegnerinnen und Gegner beschäftigen sich mit Gegenargumenten, und dies sehr selektiv, d.h. dass auch Parlamentsdebatten über die grundlegende Frage zu Krieg und Frieden ideologischen Linien und formalen Verfahrensregeln folgen.

Die wichtigsten, d.h. am häufigsten gebrauchten, Argumente gegen den Krieg, die Kriegsbefürworterinnen und Kriegsbefürworter nötigten, sich mit ihnen auseinanderzusetzen und deren Validität sie nicht bestritten, betrafen die Furcht vor hohen eigenen Opfern (15\%) und die Behauptung, die friedlichen Mittel seien noch nicht ausgeschöpft $(15,7 \%)$. Letzteres impliziert, dass das Land unwiderruflich ein militärisches Eingreifen mittragen sollte, sobald die Hoffnung auf eine friedliche Konfliktlösung endgültig begraben werden muss. Das dritthäufigste genannte Argument, allerdings mit deutlichem Abstand, ist der Verweis auf zivile Opfer der Gegenseite $(6,1 \%)$, gefolgt von dem Verweis auf das Fehlen eines UNO-Mandats $(4,4 \%)$. Die Argumentationsmuster lauteten meist, dass das Argument gegen einen Militäreinsatz als grundsätzlich bedenkenswert akzeptiert wurde, aber in einer Abwägung durch befürwortende Argumente »übertrumpft« wurde.

Kriegsgegnerinnen und Kriegsgegner beschäftigen sich vor allem mit dem Feindbild, welches die Kriegsbefürworterinnen und Kriegsbefürworter zeichnen; 15,5\% folgen diesem Feindbild durchaus. 8,3\% befürworten den Aufbau einer Drohkulisse, um die Gegenseite zum Einlenken zu zwingen, schrecken aber vor dem Einsatz der in Stellung gebrachten militärischen Fähigkeiten zurück. 6,4\% äußerten ihre Besorgnis über eine humanitäre Katastrophe (bevorzugt während des Kosovokrieges), und 5,4\% räumten ein, dass das Unterlassen einer Intervention die betroffene Region u.U. destabilisieren könnte - um dann aber jeweils mit Verweis auf in ihren Augen bedeutenderen Argumenten den Militäreinsatz als geeignetes Mittel abzulehnen.

\section{Einstieg in die Kriegseskalation: "Drohkulisse»}

Fahndet man nach dem jeweiligen »Weg in den Krieg«, so fällt auf, dass sehr viele der parlamentarischen Sprecherinnen und Sprecher bereit sind, eine militärische Drohkulisse als Druckmittel zur Erzielung von compliance zu akzeptieren. Fast 60\% $(57,4 \%)$ der Politikerinnen und Politiker, die sich für den Rückgriff auf militärische Mittel einsetzen, sehen die Entsendung eigener Truppen in die Krisenregion zum Aufbau militärischen Drucks als notwendig an und immerhin 8,3\% der Kriegsgegnerinnen und Kriegsgegner akzeptieren dies als valides Argument. Während man diese Argumentation auf den ersten Blick nicht notwendigerweise als »unfriedlich « einstufen muss, da der tatsächliche Einsatz militärischer Zwangsmittel noch offen zu sein scheint, betritt man tatsächlich aber eine schiefe Ebene (slippery slope). Die Gefahr, in einen Krieg hineinzugleiten, erhöht sich mit fortdauernder Krise stetig. 
Wie empirische Forschung zu solcher »coercive diplomacy«, deren eigentliches Ziel die Vermeidung von Krieg ist, zeigt, ist diese in den meisten Fällen gescheitert. Das unter Druck gesetzte Regime ließ sich nicht zu einer gewünschten Verhaltensänderung zwingen; stattdessen wurde eine Kette von wechselseitiger Drohpolitik, teils Fehlwahrnehmungen (über ausgesandte Signale) und Fehlspekulationen in Gang gesetzt, die die Gewalt androhenden Staaten mit hohen Kosten konfrontiert (Art/Cronin 2007). Denn sind die Truppen erst einmal in der Region vor Ort, ist ein Rückzug, ohne das gewünschte Ergebnis auf diplomatischem Wege erzielt zu haben, mit Gesichtsverlust und Verlust an Glaubwürdigkeit verbunden.

Gerade in den betrachteten Kriegen hat sich diese Zwangsdiplomatie als zweischneidiges Schwert entpuppt. Die Entscheidung für den Aufbau der Drohkulisse wird damit schneller als gewünscht zu einer Entscheidung über eine mögliche Kriegsbeteiligung. Anders ausgedrückt: Wer der Entsendung eigener Truppen zum Aufbau einer militärischen Drohkulisse zustimmt, nimmt in Kauf, dass diese Entscheidung mit einiger Wahrscheinlichkeit schon ein erster Schritt hin zum Einsatz dieser Mittel sein wird. Dieser Zusammenhang wird von einigen Abgeordneten durchaus auch so gesehen - allerdings nicht von jenen, die die Pfadabhängigkeit solcher Entsendungsentscheidungen unterschätzen und von einer stetigen Reversibilität der Entscheidung ausgehen.

\section{Die Bedeutung des Völkerrechts}

Völkerrechtlerinnen und Völkerrechtler dürften die Ergebnisse über die Rolle ihres Gegenstands im Legitimationsprozess westlicher Interventionen kaum überraschen. Alle Demokratien sehen die Notwendigkeit, ihre Politik »irgendwie« völkerrechtlich zu rechtfertigen, und zwar unabhängig davon, ob ihre Position von einer Staatenmehrheit geteilt wird, einen Bruch des geltenden Völkerrechts nach herrschender Meinung oder - euphemistisch - eine »kreative Weiterentwicklung« darstellt (Brock 2004). Angesichts dieser bedeutenden Rolle völkerrechtlicher Argumente verwundert die Vernachlässigung der Rechtsthematik in der DF-Forschung umso mehr (Liste 2009: 102). In der argumentativen Praxis erweisen sich Völkerrecht und der UNSicherheitsrat als äußerst bedeutsam für die demokratische Auseinandersetzung über die normative Rechtfertigung von Krieg und Frieden.

Der Streit in den innerstaatlichen Debatten über die völkerrechtliche Berechtigung einer Kriegsteilnahme zeigt die Ambivalenz der normativen Legitimationskriterien und die Möglichkeit widersprüchlicher Interpretationen, selbst wenn die Lage auf den ersten Blick eindeutig erscheint. Nahezu in allen Parlamentsdebatten erzeugte die Frage nach der Legitimität und Legalität eines militärischen Eingreifens eine intensive Auseinandersetzung zwischen Befürworterinnen und Befürwortern und Gegnerinnen und Gegnern. Vor dem Golfkrieg 1991, als die UN-Resolution 678 Legitimität und Legalität unmissverständlich zu etablieren schien, berief sich jede bzw. jeder zweite Befürworterin und Befürworter explizit auf die völkerrechtliche Deckung ihrer bzw. seiner Position (46,4\%), und mehr als zwei Drittel (70,6\%) vertraten die Position, den 
Buchstaben oder wenigstens dem Geist des Völkerrechts mit einem Einsatz Geltung zu verschaffen. Trotz der wesentlich umstritteneren Situation vor dem Irakkrieg 2003 behaupteten 25,4\% aller Befürworterinnen und Befürworter, dieser Krieg sei völkerrechtlich gedeckt; 41,5\% nahmen sogar in Anspruch, mit Waffen das Völkerrecht durchsetzen zu wollen. Selbst bezüglich des Kosovokriegs, der nicht von der UNO mandatiert war, argumentierten $19,7 \%$ der Befürworterinnen und Befürworter, innerhalb des völkerrechtlich Zulässigen zu handeln, während 16,1\% behaupteten, Völkerrecht direkt durchzusetzen. Auch aufseiten der Kriegsgegnerinnen und Kriegsgegner spielte das Völkerrecht eine wichtige Rolle. Selbst 1991 stellten 26,6\% von ihnen die Legalität des Krieges infrage. 1999 und 2003 betrachtete nahezu jede/r zweite Rednerin und Redner den Militäreinsatz als illegal (46,6\% bzw. 48,1\%).

Diese Befunde legen nahe, dass die Legitimität im Rahmen der Vereinten Nationen in westlichen Öffentlichkeiten hohe Anerkennung genießt und Befürworterinnen und Befürworter wie Gegnerinnen und Gegner dazu veranlasst, ihre Positionen zu Militäreinsätzen in Rechtskategorien zu »rahmen«. Allerdings zeigt die Analyse auch, dass sich ein Rechtssystem wie die UN-Charta sehr gegensätzlich interpretieren lässt. Und selbst wenn Kriegsbefürworterinnen und Kriegsbefürworter anerkennen müssen, dass ihre Position völkerrechtlich auf dünnem Boden steht, nehmen sie Zuflucht zu Hilfskonstruktionen wie dem »Geist des Völkerrechts«, dem die vorgeschriebenen Verfahren durch die obstruktive Haltung mancher Akteure keine Geltung verschaffen könnten. Trotz dieser Einschränkungen gilt indes, dass ein eindeutiges Mandat des UN-Sicherheitsrats für die Anwendung militärischer Gewalt einen sehr starken Anreiz für westliche Demokratien darstellt, an einem Militäreinsatz teilzunehmen, und dass es sehr schwierig ist, erfolgreich Argumente dagegen in Stellung zu bringen.

\section{Die Hürde der eigenen Opfer}

Die Ausnahmestellung der Opfervermeidung in der Argumentation der Befürworterinnen und Befürworter, von denen sich (s.o.) immerhin 15\% mit dieser Problematik auseinandersetzen, erfordert eine Erläuterung, zumal die Opfer der Gegenseite nur in $6,1 \%$ der Äußerungen thematisiert werden. Normative Varianten der DF-Theorie nehmen an, dass konsolidierte liberale Demokratien den Schutz des individuellen Menschenlebens ganz oben in ihrer Werteskala führen, da eine solche Wertschätzung des Individuums zu den normativen Grundlagen der politischen Kultur gehört, die in der Aufklärung wurzeln (Schörnig 2007: 96-97). Die Daten unterstützen hingegen die These einer rational inspirierten Normhierarchie, welche die Belange der eigenen Soldatinnen und Soldaten sehr deutlich vor denjenigen der Zivilistinnen und Zivilisten aufseiten des Gegners platzieren (Mandel 2004: 11; Shaw 2005: 79-83). Kommunaler Liberalismus schlägt den kosmopolitischen insofern auf dem normativen Schlachtfeld um Längen.

Allerdings zeigt der Blick auf die Kriegsgegnerinnen und Kriegsgegner, dass diese in der Tat fremde und eigene Opfer nicht nur gleichermaßen als relevant ansehen, sondern die Sorge um mögliche fremde Opfer (31,4\%) sogar häufiger artikulieren als 
die Sorge um mögliche eigene Opfer $(25,2 \%)$. Dies deutet darauf hin, dass Befürworterinnen und Befürworter eines Einsatzes die Frage potenzieller Opfer eher aus einem rationalistischen Blickwinkel sehen und gemäß einer der Bevölkerung unterstellten Normhierarchie eine »Absicherungsstrategie« betreiben (Schörnig 2007: 106), während Gegnerinnen und Gegner eines Einsatzes stärker moralische Perspektiven akzentuieren und an allgemeine Werte appellieren.

\section{Feindbild}

Wie im theoretischen Teil des Aufsatzes dargelegt, müssen die Kriegsbefürworterinnen und Kriegsbefürworter die Abneigung demokratischer Öffentlichkeiten gegen Militäreinsätze überwinden und einen neuen Konsens mit diskursiven Mitteln herstellen. Da hier die Konstruktion eines überzeugenden Feindbildes eine wichtige rhetorische Strategie darstellt (Wette 2004), sind dämonisierende Beschreibungen des Gegners durch die Befürworterinnen und Befürworter zu erwarten. Die Daten zeigen tatsächlich, dass schon im Golfkrieg 1991 mehr als ein Drittel (34,6\%) der Befürworterinnen und Befürworter eines Einsatzes auf eine starke Feindbildbeschwörung setzte. Im Vorfeld des Kosovokriegs 1999 wurde ein Feindbild Milošević nur von einem Fünftel beschworen (20,4\%). Eine starke Veränderung ergab sich im Vorfeld des Irakkrieges 2003. Fast dreiviertel (72,9\%) aller Befürworterinnen und Befürworter eines Militärschlags gegen Saddam Hussein nutzten Feindbildrhetorik, wobei besonders die Problematik von Massenvernichtungswaffen in den Händen eines ruchlosen Diktators hervorgehoben wurde und eine rhetorische Abgrenzung zu Gefahren für die eigene nationale Sicherheit verwischte.

Kontrastiert man diese Befunde mit der wesentlich schärferen Darstellung der gegnerischen Regime in einigen Medien, so scheint im Parlament ein stärkerer »Zivilisierungszwang « vorzuherrschen, der eine zu scharfe Dämonisierung des Gegners verhindert. Dies kann allerdings auch am Kodierzeitraum liegen: 1990/91 und mehr noch 1998/99 stellten die beiden Diktatoren über weite Strecken des Prozesses immer noch faktische oder potenzielle Verhandlungspartner der Demokratien dar. Eine allzu starke Verdammung dieses Gegenübers durch die politischen Entscheidungsträger hätte alle Türen zu einer diplomatischen Lösung von vornherein zugeschlagen.

\subsection{Varianz zwischen den Demokratien}

Aus einer sozialkonstruktivistischen Perspektive sind die kollektive Identität und die Rollenkonzeption eines Landes wichtige Faktoren, die die Außenpolitik bestimmen. »Realistische« Variablen wie materielle Machtressourcen rangieren demgegenüber wesentlich niedriger. Um die Varianz im außenpolitischen Verhalten erklären zu können, muss man die spezifischen Identitäten und Rollenkonzeptionen der einzelnen Staaten untersuchen. Da zu vermuten ist, dass sich nationale Identitäten und Rollenkonzeptionen in den Strukturen der nationalstaatlichen Diskurse widerspiegeln, stellt 
deren Analyse den Schlüssel dar, um die Varianz zwischen Kriegsteilnahme und -nichtteilnahme im sozialkonstruktivistischen Sinne erklären zu können.

Zunächst stellte sich die Frage, welche Länder sich in ihren Argumentationsstrukturen über alle drei Konflikte hinweg besonders »ähnlich « sind. Hierzu wurde auf die Ebene der standardisierten Häufigkeitsausprägung (»Relevanzen«, siehe Fn. 18) der einzelnen Argumente in allen Konflikten zurückgegriffen und alle 135 (3 x 45) landesspezifischen Häufigkeitsausprägungen für alle Länder korreliert. Die Stärke des Korrelationskoeffizienten wurde als Maß der Ähnlichkeit der Parlamentsdiskurse interpretiert. Dabei zeigt sich, dass Deutschland, Kanada, Schweden und Frankreich ein Ähnlichkeitscluster bilden, und dass Großbritannien und die USA einander in ihren Argumentationsstrukturen am ähnlichsten sind. Da in Australien keine Parlamentsdebatte über eine mögliche Beteiligung am Kosovokrieg stattfand, ${ }^{20}$ wurden die Ähnlichkeiten ohne Berücksichtigung der Kosovo-Debatten, aber mit Australien, erneut berechnet. Die Ergebnisse blieben im Wesentlichen stabil, einzig Großbritannien und Australien waren nun einander am ähnlichsten, während die USA weiterhin Großbritannien am ähnlichsten blieben. Schaubild 1 zeigt jeweils die größte Ähnlichkeit eines Landes mit einem anderen und die dazugehörigen Korrelationskoeffizienten auf: 21

Schaubild 1: Ähnlichkeit der Argumentationsstrukturen ${ }^{22}$

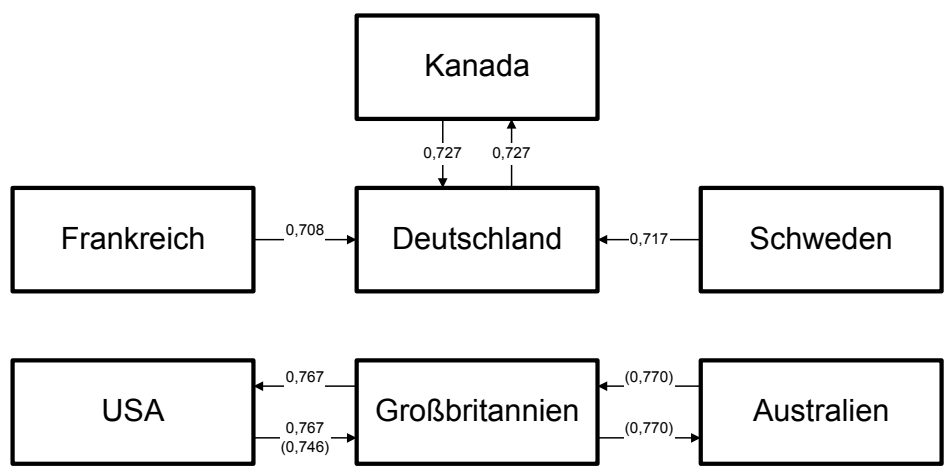

20 Informelle (und sehr zurückhaltende) Anfragen der USA und Großbritanniens wies die Regierung Howard ebenso informell zurück und vermied eine öffentliche Beteiligungsdebatte.

21 Es gilt für alle angegebenen Korrelationen $p<0.01$.

22 Die Pfeile stellen jeweils die »Richtung« der Ähnlichkeit dar: Frankreich ist z.B. Deutschland am ähnlichsten, während Deutschland selbst Kanada am ähnlichsten ist. Alle Werte ohne Klammer beziehen sich auf die Daten mit Berücksichtigung des Kosovo-Konflikts ohne Australien. Die Veränderung der Ähnlichkeitsstruktur bei Vernachlässigung des Kosovo-Konflikts und Berücksichtigung von Australien sind in Klammern angegeben. Großbritannien ist nun Australien ähnlicher als den USA. 
Der eine Block der Ähnlichkeitscluster, den Deutschland, Kanada, Schweden und Frankreich bilden, umfasst Länder, deren Kriegsbeteiligung zwischen Nichtteilnahme und selektiver Teilnahme variiert und die (mit der Ausnahme Frankreichs) in der Literatur häufig als Zivilmächte beschrieben werden. Den zweiten Ähnlichkeitscluster bilden die USA, Großbritannien und Australien. Es ist vermutlich kein Zufall, dass die US-amerikanischen, australischen und britischen Parlamentsdiskurse andere Strukturen aufweisen als die der übrigen Länder. Die USA und Großbritannien nahmen an allen drei Kriegen teil, und nur die Kombination aus geographischer Distanz und fehlendem Interesse der wichtigsten Partner USA und Großbritannien (und damit der Mangel an jeglichem nationalem Interesse) hinderte Australien an der Teilnahme am Kosovokrieg.

Im nächsten Schritt sind nun die nationalen Argumentationsstrukturen der USA, Schwedens, Deutschlands und Australiens genauer zu betrachten. Grundlage sind die $7+2$ Frames, in welche die Argumente eingehen, deren grundsätzliche Argumentationslogik bzw. deren argumentativer Bezug ähnlich ist. Schaubild 2 zeigt die USAWerte für jeden einzelnen der $7+2$ Frames in Form einer »Argumentationskarte« und gibt zum Vergleich die Durchschnittswerte der jeweiligen Frames über alle Län$\operatorname{der}^{23}$ an. ${ }^{24}$

\section{Schaubild 2: Argumentationskarte USA}

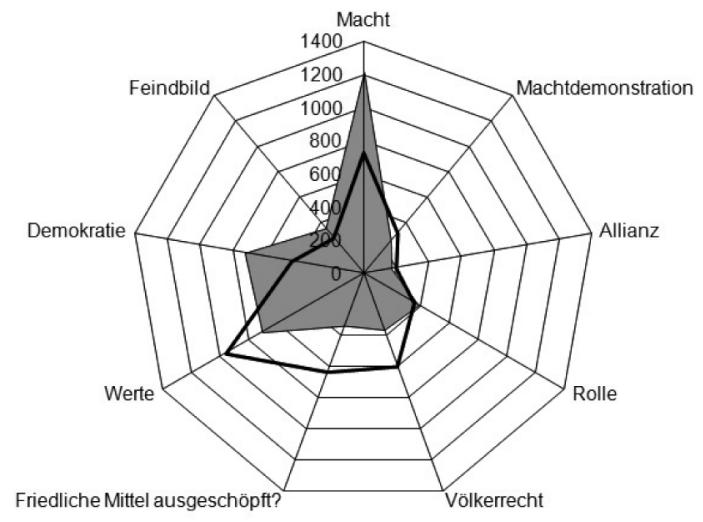

IUSA

口Durchschnitt

Man erkennt an der US-amerikanischen »Argumentationskarte«, dass in den Debatten im Kongress wesentlich stärker als in anderen Staaten auf machtbezogene Argumente rekurriert wird. »Realistische« Argumente (nationales Interesse, nationale

23 Analog der oben berechneten Ähnlichkeit wurde Australien bei der Berechnung der Durchschnittswerte nicht berücksichtigt. Die australischen Diskurse hingegen werden mit den um den Kosovo-Konflikt bereinigten Werten der anderen Staaten verglichen.

24 Es ist nochmals zu betonen, dass die absoluten Werte der Frames innerhalb eines Landes nicht miteinander verglichen werden sollten, ohne das Kategorienschema (Anhang 1) zu berücksichtigen, da unterschiedlich viele Argumente in die Frames eingehen. 
Sicherheit, regionale Stabilität) dominieren die amerikanische Debatte vor allem vor den beiden Irakkriegen, wurden aber auch bezüglich der »humanitären Intervention « im Kosovo häufiger als in allen untersuchten Demokratien angeführt, was die überdurchschnittliche Ausprägung dieses Frames erklärt. Ebenfalls deutlich überdurchschnittlich ist der Gebrauch von Argumenten, die einen Bezug zu demokratischen Normen und Prinzipien aufweisen, wie z.B. die Beachtung demokratischer Verfahren oder der verantwortungsvolle Umgang mit Steuergeldern. Besonders wichtig ist hier auch der Schutz der eigenen Truppen, worauf in praktisch jedem Konflikt mit Nachdruck hingewiesen wird. Nicht so scharf ausgeprägt, aber immer noch leicht überdurchschnittlich häufig, finden sich Hinweise auf das Rollenbild der USA, ${ }^{25}$ das die (Super-)Machtposition wie den Missionsgedanken einschließt, sowie die Konstruktion von in manichäischer Weise überspitzten Feindbildern.

Unterdurchschnittlich wird hingegen auf allgemeinere Werte verwiesen, wobei die Sorge um die bestehende politische und ökonomische Weltordnung (nicht verstanden im Sinne einer Mächteordnung im realistischen Sinn) im Rahmen der wertbezogenen Argumente noch den meisten Zuspruch erhält. Auch fallen Verweise auf drohende humanitäre Katastrophen und besonders der Schutz der »gegnerischen « Zivilbevölkerung weniger stark als in anderen Staaten ins Gewicht. Leicht unterdurchschnittlich sind Verweise auf die NATO-Allianz, wobei das Argument in der Gruppe der NATOMitglieder in den USA am wenigsten genutzt wird. Offensichtlich spielt die Allianz als Legitimationsfaktor für die Führungsnation eine geringere Rolle als für die anderen Mitglieder.

Der Frame »Völkerrecht « ist in besonderer Weise unterdurchschnittlich ausgeprägt - die USA erreichen in diesem Bereich den niedrigsten Wert aller betrachteten Staaten überhaupt. Ein Konsens im UN-Sicherheitsrat ist für die USA keine notwendige Voraussetzung für einen Militäreinsatz. Entsprechend wird auch die Frage, inwieweit die friedlichen Mittel schon ausgeschöpft seien und militärische Gewalt deshalb nun legitim sei, nur unterdurchschnittlich häufig gestellt. Wenn die Annahme zutrifft, dass Abgeordnete Argumente nutzen, von denen sie glauben, dass sie die Wählerschaft überzeugen könnten (und die daher als Ausdruck der nationalen Identität gelesen werden können), dann korrespondiert diese Argumentationsstruktur mit dem Selbstverständnis der USA als einziger Supermacht: Die USA sind aufgrund ihrer überragenden Machtposition bereit, unter Umständen auch mit ihrer überlegenden Militärgewalt ihre Interessen und (liberal-demokratischen) Werte überall in der Welt selbstbewusst zu verteidigen und zu verbreiten - vorzugsweise unterstützt durch von ihr dominierte Allianzen, mitunter aber alleine bzw. einer Koalition der Willigen, und notfalls auch verbunden mit einem Völkerrechtsbruch.

In scharfem Kontrast zur US-amerikanischen Argumentationskarte steht die schwedische - was nicht nur darauf zurückzuführen ist, dass sich das neutrale Schweden an keinem der Konflikte mit militärischen Kampftruppen beteiligte. Schweden gilt häufig als die »Zivilmacht« par excellence, und die schwedischen Parlamentsdebatten

25 Der USA-Wert liegt um 8\% über dem Durchschnitt, was bei der Auflösung der Graphik nicht angezeigt werden kann. 
unterscheiden sich (mit Ausnahme der noch relativ ähnlichen deutschen Debatten) auch deutlich von den anderen untersuchten Ländern: ${ }^{26}$

\section{Schaubild 3: Argumentationskarte Schweden}

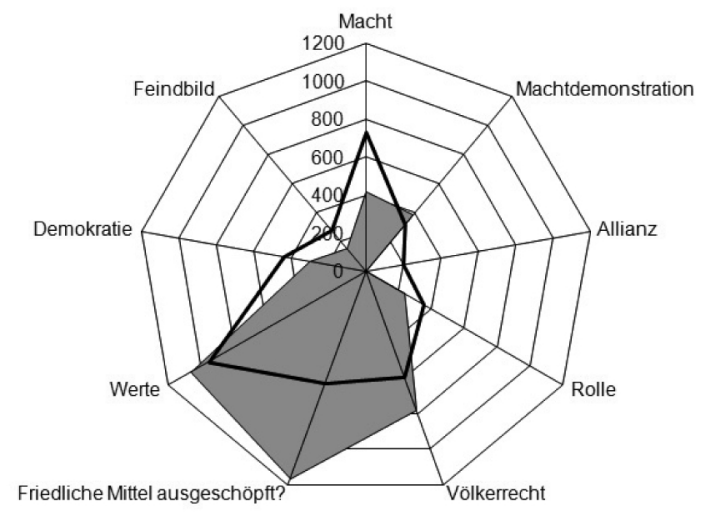

口Schweden

口Durchschnitt

Der schwedische Parlamentsdiskurs ist stark auf Völkerrecht, »friedliche Mittel « und Werte (hierbei besonders die Situation der gegnerischen Zivilbevölkerung) fokussiert, die alle weit überdurchschnittliche Ausprägungen annehmen, und entspricht damit den Erwartungen an eine Zivilmacht. Gleichwohl zeigt sich auch, dass Schweden keine pazifistische Nation ist ${ }^{27}$ - obwohl pazifistische Positionen im Diskurs durchaus artikuliert werden. Die Frage, ob die friedlichen Mittel ausgeschöpft wurden, ist für Schweden wesentlich bedeutender als für die anderen Länder; gleichzeitig können sich schwedische Abgeordnete eine militärische Machtdemonstration zur Durchsetzung bestimmter politischer Ziele durchaus vorstellen. Auch verzichten schwedische Abgeordnete nicht völlig auf die Nutzung machtbezogener Argumente oder auf ein Feindbild - sie sind dabei aber deutlich zurückhaltender als die Parlamentarierinnen und Parlamentarier in anderen Staaten.

Bei genauerer Betrachtung der Entwicklung der schwedischen Sicherheitspolitik seit 1990 zeigt sich denn auch, dass Schweden offenbar eine größere Bereitschaft zur Teilnahme an internationalen Militäroperationen entwickelt hat als gemeinhin angenommen, wobei ein UN-Mandat als die conditio sine qua non gilt (Wunderlich 2010) - weitgehend unabhängig von dem auslösenden Kriegsgrund. Liegt ein UN-Mandat vor, so ist eine Entsendung schwedischer Truppen zum Aufbau einer Drohkulisse zur Durchsetzung des Mandats vorstellbar. Ergibt sich dann noch der Eindruck, die friedlichen Mittel seien in der Tat ausgeschöpft, so kann mit einer schwedischen Kriegsbeteiligung durchaus gerechnet werden. Hinweise hierauf ergeben sich auch aus der

26 So hat Schweden den niedrigsten durchschnittlichen Korrelationskoeffizienten von allen untersuchten Ländern, also die geringste durchschnittliche Ähnlichkeit.

27 Es sei hier betont, dass das idealtypische Rollenkonzept »Zivilmacht« auch nicht gleichbedeutend mit einer »pazifistischen« Macht ist (Maull 2000: 69). 
schwedischen Haltung während des Golfkriegs 1991: Während Schweden kein offizielles Mitglied der Kriegsallianz war, verstanden und akzeptierten viele Schweden die Entsendung eines Militärhospitals dennoch als aktive Unterstützung der Kampfhandlungen am Persischen Golf.

Betrachtet man nun den Fall Deutschland, so sind Parallelen, aber auch Unterschiede zu Schweden erkennbar, wobei sich jedoch auch im deutschen Fall das außenpolitische Rollenkonzept der »Zivilmacht« in der Argumentationskarte abzeichnet:

\section{Schaubild 4: Argumentationskarte Deutschland}

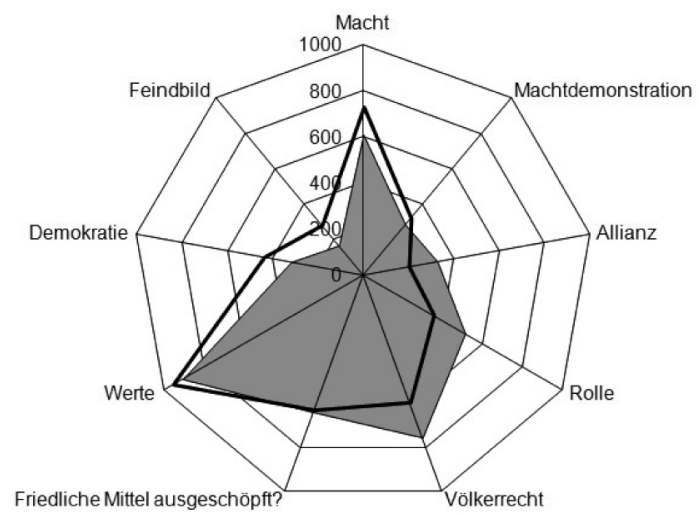

口Deutschland

口Durchschnitt

Zunächst fällt auf, dass sich die deutschen Debatten insgesamt als »durchschnittlicher« darstellen, als dies bei den beiden anderen Staaten der Fall war. Gleichwohl liegt Deutschland näher an Schweden als an den USA. Dies zeigt sich zunächst daran, dass auf Argumente mit Machtbezug in Deutschland unterdurchschnittlich häufig zurückgegriffen wird. Der Blick in die zugrunde liegenden Daten zeigt, dass diese Argumente meist benutzt werden, um gegen deutsche Militäreinsätze zu argumentieren. Ebenfalls unterdurchschnittlich häufig wird auf Feindbildkonstruktionen rekurriert - zusammen mit Schweden zeichnet sich Deutschland durch den geringsten Wert in diesem Bereich aus. Aber auch Argumente mit explizitem Demokratiebezug spielen in Deutschland eine geringere Rolle als anderswo. Wenn es um universelle Werte geht, zeigt sich Deutschland fast durchschnittlich; allerdings fanden sich im deutschen Parlamentsdiskurs im internationalen Vergleich noch die meisten pazifistischen Argumentationen (insbesondere 1990/91) und auch überdurchschnittlich häufige Verweise auf humanitäre Argumente.

Dominant im deutschen Parlamentsdiskurs sind Argumente mit Bezug auf das Völkerrecht (zweithöchster Wert nach Schweden) und das eigene Rollenverständnis; beide werden sowohl zur Legitimation als auch Zurückweisung von Militäreinsätzen benutzt. Die Frage, ob die friedlichen Mittel ausgeschöpft seien, wird zwar deutlich häufiger als z.B. in den USA artikuliert, aber ungefähr gleich häufig wie in Frankreich 
oder Kanada. Interessant ist, dass auch der Verweis auf Allianzen überdurchschnittlich häufig erfolgt, und zwar besonders dann, wenn es darum geht, einen Militäreinsatz zu legitimieren.

Insgesamt spiegelt die parlamentarische Argumentationsstruktur Deutschlands außenpolitische Konzeption als »Zivilmacht «. Im Gegensatz zu den USA werden Kriege aus »bloßen" nationalen Interessen oder aus regionalen Stabilitätsgründen in Deutschland nicht akzeptiert. Andererseits bedeutet »Zivilmacht« nicht Pazifismus (Maull 2000: 69): In einer humanitären Krise, flankiert von einem UN-Mandat oder einer gemeinsamen Entscheidung der NATO, besteht eine hohe Wahrscheinlichkeit, dass Deutschland sich einem militärischen Einsatz anschließt.

Abschließend ist nun noch der australische Fall zu betrachten:

\section{Schaubild 5: Argumentationskarte Australien}

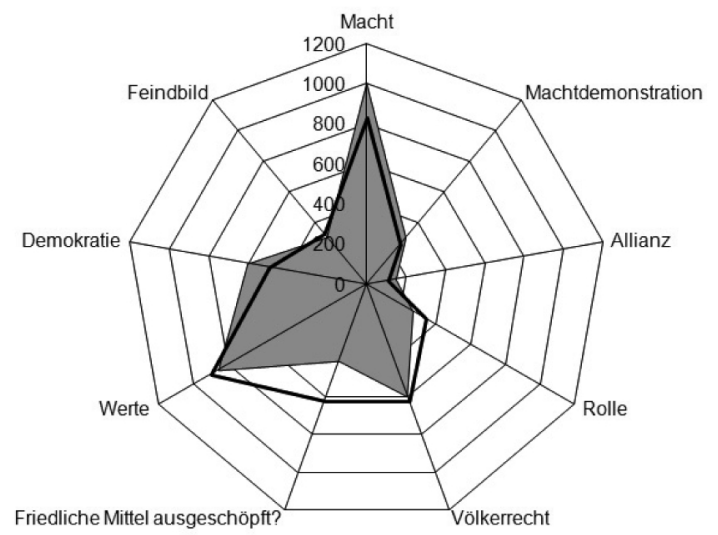

$\square$ Australien

aDurchschnitt

Im australischen Diskurs fällt zunächst besonders auf, dass Abgeordnete überdurchschnittlich häufig auf Argumente mit Machtbezug zurückgreifen, und zwar insbesondere, um einen Einsatz der Streitkräfte zu legitimieren. So wird deutlich häufiger auf das nationale Interesse oder die nationale Sicherheit verwiesen, als dies in den meisten anderen Staaten der Fall ist. Ebenfalls überdurchschnittlich häufig rekurrieren australische Abgeordnete auf demokratische Werte, wobei die Sorge um die eigenen Soldaten eine besondere Rolle spielt. Aber auch Verweise auf die öffentliche Meinung als von den politischen Eliten zu berücksichtigender Faktor finden sich in den Debatten überdurchschnittlich häufig.

Verweise auf allgemeine Werte finden sich dagegen durchschnittlich häufig, wobei hier besonders die Sorge um die bestehende politische und ökonomische Weltordnung betont wird. Absolut gesehen nicht sonderlich häufig, aber verglichen mit anderen westlichen Staaten immer noch überdurchschnittlich, sind Bezüge auf die Allianz mit den USA, die von den australischen Eliten als sicherheitspolitisch sehr bedeutend wahrgenommen wird. Leicht unterdurchschnittlich sind im australischen Diskurs Re- 
ferenzen auf die eigene Rolle oder die nationale Identität zu finden sowie die Frage, ob die friedlichen Mittel erschöpft seien. Argumente, die auf das Völkerrecht verweisen, sind im Vergleich zu anderen Demokratien durchschnittlich häufig vertreten.

In diesem Argumentationsmuster schlägt sich Australiens besondere Identität und Rollenkonzeption nieder, in der die »realistischen « Komponenten nationales Interesse und nationale Sicherheit mit dem zumindest in Teilen der Eliten vorhandenen Ehrgeiz, ein »guter internationaler Bürger « zu sein, koexistieren. Aus australischer Perspektive ist dies kein Widerspruch. Es liegt im australischen Interesse, das Völkerrecht zu achten und die Weltordnung zu stützen - und wenn nötig, beide Interessen auch mit Gewalt gegen böswillige Gegner durchzusetzen. Australierinnen und Australier gehen davon aus, sich selbst in einer relativ instabilen Region zu befinden, in denen Gefährdungen für die politische Ordnung oder Verletzungen des Völkerrechts drohen. Im australischen Verständnis wird die rechtsgestützte Weltordnung daher weniger stark normativ aufgeladen betrachtet, als dies etwa in Schweden der Fall ist; sie wird stattdessen in einem stärker instrumentellen Sinne geschätzt, um das Überleben des Landes in einer gefährlichen Umgebung zu sichern.

Insgesamt zeigt die Analyse der - wenn auch sehr groben - »Argumentationskarten «, dass die nationalen Parlamentsdiskurse in weiten Bereichen die kollektiven Identitäten der Länder widerspiegeln, wie sie häufig in der Sekundärliteratur beschrieben werden. Die Sprecherinnen und Sprecher greifen in der Tat auf Argumente (bzw. Argumentationslogiken) zurück, die in den nationalen Idiosynkrasien besondere Resonanz erzeugen können.

\section{Schlussfolgerungen für den »demokratischen Frieden« und den »demokratischen Krieg"}

Was lässt sich nun aus den Daten und den umfassenden Fallstudien, auf denen sie beruhen (Geis et al. 2010), für die Forschung zum demokratischen Frieden und demokratischen Krieg schließen?

\section{Demokratische Debatten über die Anwendung militärischer Gewalt}

Erstens unterstützen die Analysen der öffentlichen Vorkriegsdiskurse in liberalen Demokratien einige grundlegende Annahmen monadischer DF-Theorie: dass konsolidierte Demokratien nicht »kriegsbegeistert« sind, dass größere militärische Aktionen einer öffentlichen Rechtfertigung bedürfen und dass der Gewalteinsatz heftig umstritten bleibt. Die meisten politischen Akteure in Demokratien betrachten kriegerische Handlungen als ultima ratio liberaler Weltordnungspolitik. Diese Aussage muss allerdings modifiziert werden: Wann die friedlichen Mittel tatsächlich erschöpft sind, ist ein praktisches Urteil, das in der politischen Debatte heftig umkämpft ist. Außerdem akzeptieren viele demokratische Politiker die militärische Machtdemonstration als eine legitime Unterstützung diplomatischer Bemühungen - nehmen damit 
aber, oft unbewusst und unabsichtlich, die Eskalation in den Krieg in Kauf. Es ist zudem auffällig, dass die zentrale Frage, ob die friedlichen Mittel der Konfliktlösung ausgeschöpft seien, in den USA weniger Berücksichtigung fand als in allen anderen untersuchten Demokratien.

Bemerkenswert ist die relativ hohe Konvergenz zwischen Elitenpositionen und öffentlicher Meinung: Die Eliten legten eine weite Palette von Rechtfertigungen für die Teilnahme an militärischen Operationen vor, und in zehn von 13 analysierten Fällen der Kriegsbeteiligung unterstützte die Öffentlichkeit laut nationaler Umfragen die Beteiligung mehrheitlich, obgleich es sich in keinem der Fälle unzweifelhaft um Selbstverteidigung handelte. Es wäre daher ein Fehler, eine a-priori-Immunität demokratischer Öffentlichkeiten gegen militärische Gewaltanwendung zu unterstellen, wie es in der monadischen DF-Theorie häufig geschieht (Hils 2008). Zugleich wird daran deutlich, dass in drei Fällen Regierungen sich für den Kriegseinsatz entschieden, obgleich ihre Anstrengungen, die Bevölkerung zu überzeugen, vergeblich waren. Hier zeigen sich Grenzen der Wirksamkeit demokratischer Kontrolle: Regierungen vertrauen darauf, dass bei der nächsten Wahl der Wählerschaft andere Belange wichtiger sein werden als die unerwünschte Beteiligung an einem räumlich - und mittlerweile vielleicht auch zeitlich - fernen Krieg; oder die Motive der Exekutive für die Anwendung militärischer Gewalt sind derart stark, dass auch der drohende Verlust einer Wahl keine hemmende Wirkung zeigt.

\section{Ein »demokratischer Krieg»?}

Im Einklang mit einer Reihe von Studien über den »liberalen Interventionismus « war eine Tendenz liberaler Demokratien erwartbar, ihre zunehmenden militärischen Interventionen und Kriege mit liberalen Werten, Normen und Weltordnungsmodellen zu rechtfertigen. Wie die Daten zeigen, war diese Erwartung richtig, aber unvollständig. Gerade in den Ländern mit relativ häufiger Kriegsteilnahme verbinden sich solche liberalen Gründe mit eher klassisch-realistischen und auf Macht bezogenen Rechtfertigungen wie nationalem Interesse, regionale Stabilität und nationale Sicherheit. Dieser Frame ist nicht demokratiespezifisch. Typisch für Demokratien ist allerdings die - in manchen Fällen anscheinend unauflösliche - Verknüpfung mit Elementen des liberalen Denkens.

Begreift man das Konzept des »demokratischen Krieges« als einen Militäreinsatz, der ausschließlich mit liberalen Gründen gerechtfertigt wird, so bliebe dieser Kriegstyp ein Idealtyp. »Reine demokratische Kriege« in diesem Sinne zeigen die hier analysierten Diskurse in der Regel nicht; häufig verbinden sich dagegen traditionelle Rechtfertigungen mit liberalen Normen - aber diese Mischung selbst kann eben auch als »demokratiespezifisch « gelten. Eine interessante Ausnahme bildet der deutsche Diskurs zum Kosovokrieg: Obwohl traditionelle Motive (eine weitere Destabilisierung des Balkans zu verhindern und die Flüchtlingsströme einzudämmen) zweifellos 
eine Rolle spielten, traten sie in der parlamentarischen Debatte kaum auf. Die Abwesenheit »realistischer« Sprache ist typisch für Deutschland. ${ }^{28}$

An dieser Stelle stellt sich die Frage, was »demokratiespezifisch « ist; denn der durchgeführte Vergleich hat ja nur Debatten in Demokratien einbezogen. ${ }^{29}$ Der Vergleich mit Nichtdemokratien wäre der logische nächste Schritt, stößt aber auf Quellenprobleme. Einschlägige Zeitungsartikel, Parlamentsdebatten und Umfragen finden sich in Nichtdemokratien zumeist nicht in der gleichen Dichte und Authentizität wie in demokratischen Umfeldern. Aus diesem Grund scheinen vorläufig reine Plausibilitätsüberlegungen möglich: Welche Argumente sind in Nichtdemokratien eher unwahrscheinlich? So sollten etwa Argumente, die sich auf demokratische Verfahren, auf demokratischen Regimewandel, auf den Schutz von Menschenrechten, auf Demokratieelemente in der eigenen Identität oder Rollenkonzeption oder auf ein Feindbild stützen, das gerade durch den nichtdemokratischen Charakter des Gegners beschrieben wird, typischerweise eher in liberalen Demokratien aufzufinden sein.

\section{Umstrittene Normen}

Die »Mischform« der von den Demokratien geführten Kriege, in deren Motivation sich spezifisch liberale Zielsetzungen und realpolitische nationale Interessen verquicken, impliziert nicht, dass diese militärischen Konflikte Anomalien für die DFTheorie darstellen. Wie im Konzept der »Antinomien « der Demokratischen-FriedensTheorie (Abschnitt 2.1) vermutet, zeigte sich, dass all jene Erklärungen, die gemäß der DF-Theorie die Friedfertigkeit der Demokratien bedingen, grundsätzlich in die gegenläufige Richtung wirken können. ${ }^{30}$ Kosten/Nutzen-Kalkulationen, internationale Institutionen und liberal-demokratische Normen und Werte fördern nicht nur friedliches Verhalten, sondern dienen in Rechtfertigungsdiskursen auch als Begründungen für militärische Aktionen. Diese Befunde zeigten sich am deutlichsten in der Wirkung und Handhabung innerer und internationaler Normen: Kulturell-normative Erklärungen des DF enthalten die »produktivsten« Antinomien, d.h. diejenigen, die am deutlichsten ihre gegensätzlichen Wirkungen entfalten. Sozialkonstruktivistische Ansätze sind daher besonders hilfreich, die Teilnahme bzw. Nichtteilnahme der Länderauswahl an militärischen Operationen zu beleuchten - selbstredend ohne hier eine komplette Erklärung beanspruchen zu können oder zu wollen. Die einzelnen Fallstudien ergaben, dass diejenigen Normen, welche die nationale Identität und die außenpolitische Rollenkonzeption strukturieren, die Kriegs(nicht)teilnahme zu einem großen Teil erklären können.

Der Vergleich der Fälle legt ferner nahe, dass die länderspezifische Art und Weise, die breite, ambivalente Bedeutung der liberalen Normen zu verengen, ein Schlüssel

28 »The Germans have eliminated the concept of >power They speak the language of >political responsibility< instead« (Katzenstein 1997: 2).

29 Vgl. hierfür ausführlicher die kontroverse Debatte zwischen Evangelista/Reppy (2008) und Müller/Schörnig (2008).

30 Zur Umstrittenheit von Normen in einem anderen Kontext siehe Wiener (2008: Kap. 3, 4). 
zum Verständnis der erheblichen Unterschiede zwischen den sieben Demokratien darstellt. Wie die demokratischen Öffentlichkeiten jeweils die eigene Positionierung in der internationalen Machtstruktur, die Allianzverpflichtungen, die Interpretation völkerrechtlicher Normen, die wünschenswerte Weltordnung und die signifikanten »Anderen« deuten, unterscheidet sich zwischen den westlichen Demokratien ganz erheblich. Die politisch verbreitete Rede von der »Gemeinschaft westlicher Demokratien« als kohärenter Wertegemeinschaft ist daher zu relativieren. Selbst in einer so grundlegenden Frage wie »Krieg oder Frieden« besteht eine weitreichende Differenzierung der Geltung von Normen, ihrer Interpretationen und zahlreiche unterschiedliche Urteile über die Angemessenheit militärischer Mittel. Differenzen, die sowohl die Diskurse innerhalb wie zwischen den Demokratien prägen.

\section{Literatur}

Art, Robert J./Cronin, Patrick M. 2007: Coercive Diplomacy, in: Crocker, Chester A./Hampson, Fen Osler/Aall, Pamela (Hrsg.): Leashing the Dogs of War, Washington, D.C., 299-318.

Auerswald, David P. 1999: Inward Bound: Domestic Institutions and Military Conflicts, in: International Organization 53: 3, 469-504.

Baumann, Rainer 2006: Der Wandel des deutschen Multilateralismus, Baden-Baden.

Benoit, Kenneth J. 1996: Democracies Really Are More Pacific (In General), in: Journal of Conflict Resolution 40: 4, 636-657.

Blair, Tony 1999: Doctrine of the International Community (Rede im Economic Club, Chicago, 24.4.1999), in: http://www.number10.gov.uk/Page1297; 13.1.2010.

Boekle, Henning/Rittberger, Volker/Wagner, Wolfgang 2001: Constructivist Foreign Policy Theory, in: Rittberger, Volker (Hrsg.): German Foreign Policy Since Unification, Manchester, 105-37.

Brock, Lothar 2004: Frieden durch Recht (HSFK-Standpunkte Nr. 3/2004), Frankfurt a. M.

Brock, Lothar 2007: Universalismus, politische Heterogenität und ungleiche Entwicklung, in: Geis/Müller/Wagner 2007, 45-67.

Brock, Lothar/Geis, Anna/Müller, Harald 2006: The Case for a New Research Agenda: Explaining Democratic Wars, in: Geis/Brock/Müller 2006, 195-214.

Bueno de Mesquita, Bruce/Morrow, James D./Siverson, Randolph M./Smith, Alastair 1999: An Institutional Explanation of the Democratic Peace, in: American Political Science Review 93: 4, 791-807.

Chafetz, Glenn/Spirtas, Michael/Frankel, Benjamin 1999: Introduction: Tracing the Influence of Identity on Foreign Policy, in: Security Studies 8: 2-3, vii-xxii.

Chojnacki, Sven 2006: Democratic Wars and Military Interventions, 1946-2002, in: Geis/Brock/ Müller 2006, 13-39.

Czempiel, Ernst-Otto 1996: Kants Theorem. Oder: Warum sind die Demokratien (noch immer) nicht friedlich?, in: Zeitschrift für Internationale Beziehungen 3: 1, 79-101.

Daase, Christopher 2004: Demokratischer Frieden - Demokratischer Krieg, in: Schweitzer, Christine/Aust, Björn/Schlotter, Peter (Hrsg.): Demokratien im Krieg, Baden-Baden, 53-71.

Deitelhoff, Nicole 2009: The Business of Security and the Transformation of the State, Universität Bremen: TranState Paper Nr. 87/2009.

Dembinski, Matthias/Hasenclever, Andreas/Freistein, Katja/Weiffen, Britta/Yamauchi, Makiko 2009: Managing Rivalries - Regional Security Institutions and Democracy in Western Europe, South America, Southeast Asia and East Asia, Osnabrück: Forschung DSF Nr. 22. 
Dieterich, Sandra/Hummel, Hartwig/Marschall, Stefan 2009: »Kriegsspielverderber?« Europäische Parlamente und der Irakkrieg 2003, in: Zeitschrift für Internationale Beziehungen 16: 1, 5-38.

Dixon, William/Senese, Paul 2002: Democracy, Disputes, and Negotiated Settlements, in: Journal of Conflict Resolution 46: 4, 547-71.

Duffield, John 1999: Political Culture and State Behavior: Why Germany Confounds Neorealism, in: International Organization 53: 4, 170-198.

Eberl, Oliver/Fischer-Lescano, Andreas 2005: Grenzen des demokratischen Rechts? Die Entsendeentscheidungen zum Irakkrieg in Großbritannien, den USA und Spanien (HSFKReport Nr. 8), Frankfurt a. M.

Evangelista, Matthew/Reppy, Judith 2008: Much Ado About Democracy: Some Sceptical Observations on Democracies and War, in: Evangelista, Matthew/Müller, Harald/Schörnig, Niklas (Hrsg.): Democracy and Security, London, 167-186.

Freedman, Lawrence 2005: The Age of Liberal Wars, in: Armstrong, David/Farrell, Theo/Maiguashca, Bice (Hrsg.): Force and Legitimacy in World Politics, Cambridge, 93-107.

Freedman, Lawrence 2006/7: Iraq, Liberal Wars and Illiberal Containment, in: Survival 48: 4, 51-65.

Geis, Anna 2001: Diagnose: Doppelbefund - Ursache ungeklärt? Die Kontroversen um den »demokratischen Frieden«, in: Politische Vierteljahresschrift 42: 2, 283-298.

Geis, Anna/Brock, Lothar/Müller, Harald (Hrsg.) 2006: Democratic Wars: Looking at the Dark Side of Democratic Peace, Houndmills.

Geis, Anna/Müller, Harald/Wagner, Wolfgang (Hrsg.) 2007: Schattenseiten des Demokratischen Friedens. Zur Kritik einer Theorie liberaler Außen- und Sicherheitspolitik, Frankfurt a. M.

Geis, Anna/Müller, Harald/Schörnig, Niklas (Hrsg.) 2010: The Janus Face of Liberal Democracies: Militant »Forces for Good» (derzeit unter Begutachtung).

Geis, Anna/Wagner, Wolfgang 2011: How Far Is It from Königsberg to Kandahar? Democratic Peace and Democratic Violence in International Relations, in: Review of International Studies, i.E.

Gleditsch, Nils Petter/Christiansen, Lene/Hegre, Håvard 2007: Democratic Jihad? Military Intervention and Democracy (World Bank Policy Research Paper WP 4242), Washington, D.C.

Harnisch, Sebastian/Maull, Hanns W. 2001: »Learned its lessons well?«: Germany as a Civilian Power Ten Years After Unification, in: Harnisch, Sebastian/Maull, Hanns W. (Hrsg.): Germany as a Civilian Power, Manchester, 128-156.

Hasenclever, Andreas 2003: Liberale Ansätze zum »demokratischen Frieden«, in: Schieder, Siegfried/Spindler, Manuela (Hrsg.): Theorien der Internationalen Beziehungen, Opladen, 199-226.

Hellmann, Gunther, unter Mitarbeit von Rainer Baumann und Wolfgang Wagner 2006: Deutsche Außenpolitik. Eine Einführung, Wiesbaden.

Hils, Jochen 2008: Der »demokratische Krieg« als Folge verfälschter Präferenzbildung?, in: Zeitschrift für Internationale Beziehungen 15: 2, 237-271.

Ish-Shalom, Piki 2006: Theory as a Hermeneutical Mechanism: The Democratic-Peace Thesis and the Politics of Democratization, in: European Journal of International Relations 12: 4, 565-599.

Kahl, Colin H. 1999: Constructing a Separate Peace: Constructivism, Collective Liberal Identity, and Democratic Peace, in: Security Studies 8: 2-3, 94-144.

Kantner, Cathleen/Kutter, Amelie/Renfordt, Swantje 2008: The Perception of the EU as an Emerging Security Actor in Media Debates on Humanitarian and Military Interventions 1990-2006 (RECON Online Working Paper 2008/19), Oslo.

Katzenstein, Peter 1996: Introduction, in: Katzenstein, Peter (Hrsg.): The Culture of National Security, New York, NY, 1-26.

Katzenstein, Peter 1997: United Germany in an Integrating Europe, in: Katzenstein, Peter (Hrsg.): Tamed Power. Germany in Europe, Ithaca, NY, 1-48. 
Kennedy, David 1997: Culture Wars: The Sources and Uses of Enmity in American History, in: Fiebig-von Hase, Ragnhild/Lehmkuhl, Ursula (Hrsg.): Enemy Images in American History, Oxford, 339-356.

Kirste, Knut/Maull, Hanns W. 1996: Zivilmacht und Rollentheorie, in: Zeitschrift für Internationale Beziehungen 3: 2, 283-312.

Lipson, Charles 2003: Reliable Partners, Princeton, NJ.

Liste, Philip 2009: Völkerrecht-Sprechen. Zur Konstruktion demokratischer Völkerrechtspolitik am Beispiel der Vereinigten Staaten von Amerika und der Bundesrepublik Deutschland. Dissertationsschrift, Frankfurt a. M.

MacMillan, John 2003: Beyond the Separate Peace, in: Journal of Peace Research 40: 2, 233-243.

Mandel, Robert 2004: Security, Strategy, and the Quest for Bloodless War, Boulder, CO/London.

Maull, Hanns W. 2000: Zivilmacht Deutschland, in: Senghaas, Dieter (Hrsg.): Frieden machen, Frankfurt a. M., 63-76.

Mearsheimer, John J. 2001: The Tragedy of Great Power Politics, New York, NY.

Müller, Harald 2002: Antinomien des demokratischen Friedens, in: Politische Vierteljahresschrift 43: 1, 46-81.

Müller, Harald 2004: The Antinomy of Democratic Peace, in: International Politics 41: 4, 494-520.

Müller, Harald 2007: Vorüberlegungen zu einer Theorie der Ambivalenz liberal-demokratischer Außen- und Sicherheitspolitik, in: Geis/Müller/Wagner 2007, 287-312.

Müller, Harald/Becker, Una 2008: Technology, Nuclear Arms Control, and Democracy, in: Evangelista, Matthew/Müller, Harald/Schörnig, Niklas (Hrsg.): Democracy and Security, London, 102-119.

Müller, Harald/Schörnig, Niklas 2008: Security Studies' Cinderella? Why Democratic Peace Theory Should be Invited to the King's Ball, in: Evangelista, Matthew/Müller, Harald/ Schörnig, Niklas (Hrsg.): Democracy and Security, London, 187-207.

Müller, Harald/Wolff, Jonas 2006: Many Data, Little Explanation, in: Geis/Brock/Müller 2006, 41-73.

O'Hanlon, Michael 2000: Technological Change and the Future of Warfare, Washington, D.C.

Peters, Dirk/Wagner, Wolfgang 2009: Revisiting Reverse Causality: External Threat and the Parliamentary Control of Military Missions: Papier für die Jahrestagung der ISA, 15.-18. Februar 2009, New York, NY.

Reiter, Dan/Stam, Allan C. 2002: Democracies at War, Princeton, NJ.

Risse, Thomas 2000: »Let's Argue!« Communicative Action in World Politics, in: International Organization 54:1, 1-39.

Risse-Kappen, Thomas 1995: Democratic Peace - Warlike Democracies? A Social Constructivist Interpretation of the Liberal Argument, in: European Journal of International Relations 1: 4, 491-517.

Rummel, Rudolph R. 1995: Democracies ARE Less Warlike Than Other Regimes, in: European Journal of International Relations 1: 4, 457-479.

Russett, Bruce 1993: Grasping the Democratic Peace, Princeton, NJ.

Russett, Bruce/Oneal, John R. 2001: Triangulating Peace: Democracy, Interdependence, and International Organizations, New York, NY.

Schimmelfennig, Frank 2001: The Community Trap: Liberal Norms, Rhetorical Action, and the Eastern Enlargement of the EU, in: International Organization 55:1, 47-80.

Schörnig, Niklas 2007: Visionen unblutiger Kriege, in: Geis/Müller/Wagner 2007, 93-121.

Schwab-Trapp, Michael 2002: Kriegsdiskurse, Opladen.

Shaw, Martin 2005: The New Western Way of War, London.

Singer, Peter W. 2009: Wired for War, New York, NY.

Smith, Tony 2007: A Pact with the Devil, New York, NY.

Spanger, Hans-Joachim/Wolff, Jonas 2007: Universales Ziel - partikulare Wege? Externe Demokratieförderung zwischen einheitlicher Rhetorik und vielfältiger Praxis, in: Geis/Müller/Wagner 2007, 261-284. 
Stahl, Bernhard 2006: Frankreichs Identität und außenpolitische Krisen, Baden-Baden.

Wagner, Wolfgang 2006: Parliamentary Control of Military Missions: Accounting for Pluralism, Genf: DCAF Occasional Paper Nr. 12.

Wendt, Alexander 1999: Social Theory of International Politics, Cambridge.

Wette, Wolfram 2004: Saddam Hussein als Wiedergänger Hitlers? in: Schweitzer, Christine/ Aust, Björn/Schlotter, Peter (Hrsg.): Demokratien im Krieg, Baden-Baden, 109-126.

Wiener, Antje 2004: Contested Compliance: Interventions on the Normative Structures of World Politics, in: European Journal of International Relations 10: 2, 189-234.

Wiener, Antje 2008: The Invisible Constitution of Politics, Cambridge.

Wunderlich, Carmen 2010: Moving Beyond Neutrality: Sweden's Changing Attitude Towards the Military Use of Force, in: Geis/Müller/Schörnig 2010 (derzeit unter Begutachtung).

Zangl, Bernhard/Zürn, Michael 2003: Frieden und Krieg, Frankfurt a. M. 
Anhang 1: Kategorienschema der Inhaltsanalyse

\begin{tabular}{|c|c|c|c|c|c|c|c|}
\hline 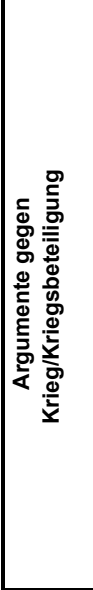 & 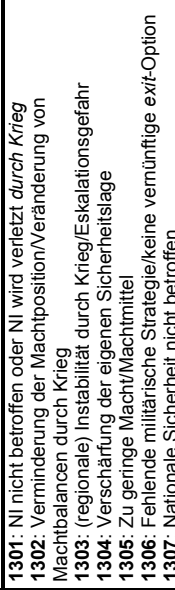 & 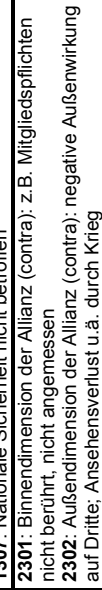 & 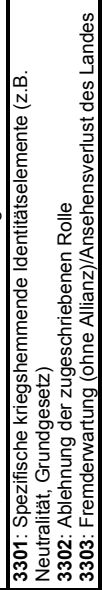 & 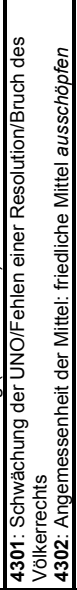 & 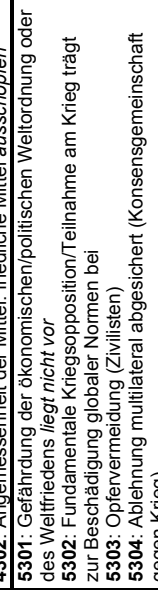 & 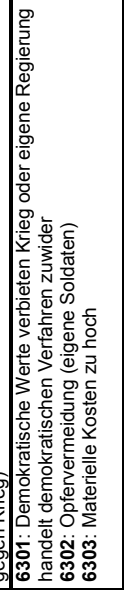 & 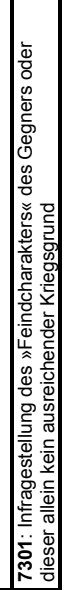 \\
\hline 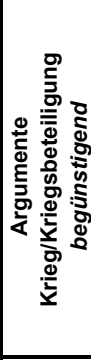 & 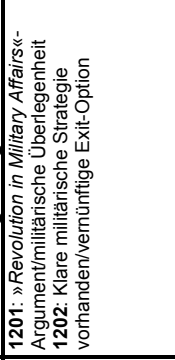 & 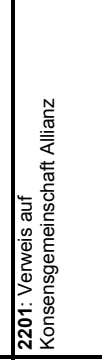 & 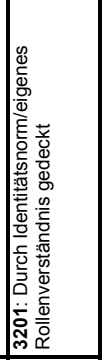 & 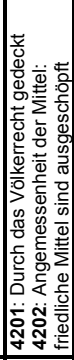 & 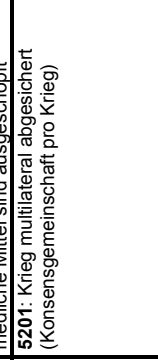 & 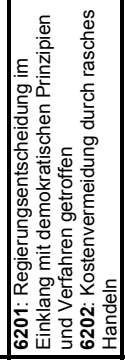 & 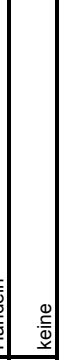 \\
\hline 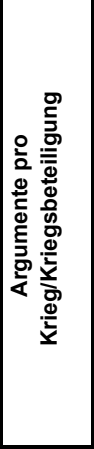 & 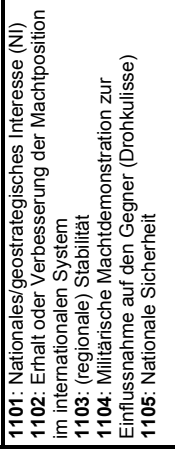 & 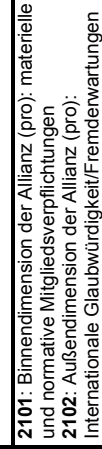 & 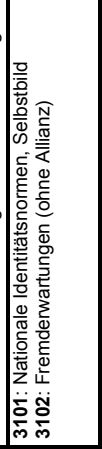 & 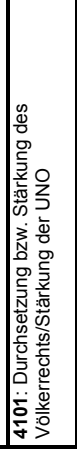 & 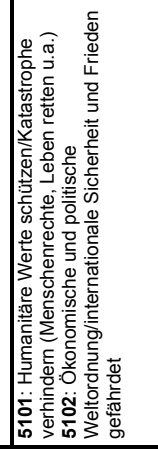 & 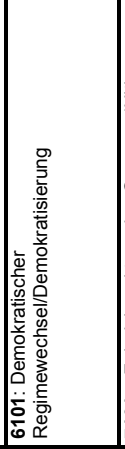 & 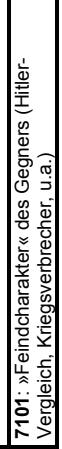 \\
\hline 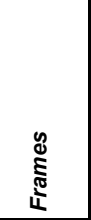 & $\begin{array}{l}\bar{\delta} \\
\Phi \\
\Sigma\end{array}$ & $\bar{\gamma}$ & 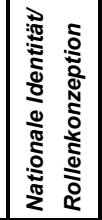 & ঃ & 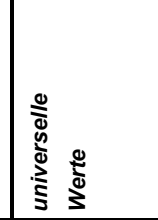 & 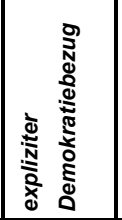 & 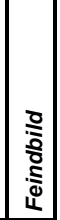 \\
\hline
\end{tabular}

